\title{
Genomic surveillance for hypervirulence and multi-drug resistance in invasive Klebsiella pneumoniae from South and Southeast Asia
}

Kelly L. Wyres ${ }^{1}$, To N. T. Nguyen², Margaret M. C. Lam ${ }^{1}$, Louise M. Judd', Nguyen van Vinh Chau' ${ }^{3}$, David A. B. Dance ${ }^{4,5,6}$, Margaret Ip ${ }^{7}$, Abhilasha Karkey, ${ }^{5,8}$, Clare L. Ling ${ }^{5,9}$, Thyl Miliya ${ }^{10}$, Paul N. Newton ${ }^{4,5,6}$, Nguyen Phu Huong Lan ${ }^{3}$, Amphone Sengduangphachanh" ${ }^{4}$ Paul Turner ${ }^{5,10}$, Balaji Veeraraghavan ${ }^{11}$, Phat Voong Vinh ${ }^{2}$, Manivanh Vongsouvath ${ }^{4}$, Nicholas R. Thomson ${ }^{6,12}$, Stephen Baker ${ }^{13^{*}}$ (D) and Kathryn E. Holt ${ }^{1,6}$

\begin{abstract}
Background: Klebsiella pneumoniae is a leading cause of bloodstream infection (BSI). Strains producing extendedspectrum beta-lactamases (ESBLs) or carbapenemases are considered global priority pathogens for which new treatment and prevention strategies are urgently required, due to severely limited therapeutic options. South and Southeast Asia are major hubs for antimicrobial-resistant (AMR) K. pneumoniae and also for the characteristically antimicrobial-sensitive, community-acquired "hypervirulent" strains. The emergence of hypervirulent AMR strains and lack of data on exopolysaccharide diversity pose a challenge for $K$. pneumoniae BSI control strategies worldwide.
\end{abstract}

Methods: We conducted a retrospective genomic epidemiology study of 365 BSI K. pneumoniae from seven major healthcare facilities across South and Southeast Asia, extracting clinically relevant information (AMR, virulence, $K$ and O antigen loci) using Kleborate, a K. pneumoniae-specific genomic typing tool.

Results: K. pneumoniae BSI isolates were highly diverse, comprising 120 multi-locus sequence types (STs) and 63 Kloci. ESBL and carbapenemase gene frequencies were $47 \%$ and $17 \%$, respectively. The aerobactin synthesis locus (iuc), associated with hypervirulence, was detected in $28 \%$ of isolates. Importantly, $7 \%$ of isolates harboured iuc plus ESBL and/or carbapenemase genes. The latter represent genotypic AMR-virulence convergence, which is generally considered a rare phenomenon but was particularly common among South Asian BSI (17\%). Of greatest concern, we identified seven novel plasmids carrying both iuc and AMR genes, raising the prospect of co-transfer of these phenotypes among K. pneumoniae.

Conclusions: K. pneumoniae BSI in South and Southeast Asia are caused by different STs from those predominating in other regions, and with higher frequency of acquired virulence determinants. K. pneumoniae carrying both iuc and AMR genes were also detected at higher rates than have been reported elsewhere. The study demonstrates how genomics-based surveillance-reporting full molecular profiles including STs, AMR, virulence and serotype locus information — can help standardise comparisons between sites and identify regional differences in pathogen populations.

Keywords: Klebsiella pneumoniae, Bloodstream infection, MDR, Capsule types, Hypervirulent, Genomic surveillance

\footnotetext{
* Correspondence: sgb47@medschl.cam.ac.uk

${ }^{13}$ Cambridge Institute of Therapeutic Immunology \& Infectious Disease

(CITIID) Department of Medicine, University of Cambridge, Cambridge

Biomedical Campus, Cambridge CB2 OAW, UK

Full list of author information is available at the end of the article
}

(c) The Author(s). 2020 Open Access This article is distributed under the terms of the Creative Commons Attribution 4.0 International License (http://creativecommons.org/licenses/by/4.0/), which permits unrestricted use, distribution, and reproduction in any medium, provided you give appropriate credit to the original author(s) and the source, provide a link to the Creative Commons license, and indicate if changes were made. The Creative Commons Public Domain Dedication waiver (http://creativecommons.org/publicdomain/zero/1.0/) applies to the data made available in this article, unless otherwise stated. 


\section{Background}

Klebsiella pneumoniae is regarded globally by the World Health Organization (WHO) as a priority antimicrobialresistant (AMR) pathogen requiring new control strategies [1]. These include rapid identification and containment of high-risk AMR clones such as the carbapenemaseproducing $(\mathrm{CP})$ variants, augmented with vaccines, bacteriophages, or immunotherapies that target surface antigens. However, K. pneumoniae is highly diverse, hindering the development of such strategies and our ability to study its molecular epidemiology in a relevant time-frame.

This diverse bacterial species is generally associated with a range of differing community and healthcareassociated infections, but can be particularly problematic when the organisms gain access to sterile sites such as the cerebrospinal fluid and the bloodstream. Such infections are often characterised by rapid onset and multidrug resistance (MDR), including resistance to thirdgeneration cephalosporins and/or carbapenems. Antimicrobials are the primary treatment strategy but options can be severely limited by AMR, particularly for thirdgeneration cephalosporin- and carbapenem-resistant strains causing invasive infections [2]. Concomitant with this are elevated mortality rates and treatment costs [3].

$K$. pneumoniae is among the most common cause of bloodstream infections (BSI) in South (S) and Southeast (SE) Asia [4-6] where it is associated with a high mortality rate $[4,7]$. Available data suggest a heterogenous landscape in terms of drug resistance; for example, CP strains are rare in SE Asia $(<1-4 \%[5,8,9])$ but common in $\mathrm{S}$ Asia $(28-70 \%[10,11])$ and the prevalence of extendedspectrum beta-lactamase (ESBL, confers resistance to the third-generation cephalosporins) producing organisms varies from 12 to $79 \%$ in these regions $[4-6,8,10]$. Studies investigating ESBL and $\mathrm{CP}$ variants in S/SE Asia implicate CTX-M-15 as the most common ESBL type [12, 13], while NDM and OXA-48-like enzymes are the most commonly described carbapenemases [11, 14-16]. However, there is currently only limited information about the underlying population structure of these organisms in terms of multilocus sequence types (STs), or genomically defined phylogenetic lineages.

Searching PubMed for reports of multi-locus sequence typing (MLST) or whole-genome sequence analysis of $K$. pneumoniae BSI (see "Methods" for search terms) yielded just nine studies reporting ST information for India, Nepal, Vietnam, Thailand, Laos, Cambodia and/or Hong Kong (the foci of this work). The majority of these (six of nine) were case reports or genome announcements from India, while an additional study from India described a screen of Enterobacteriaceae isolated from neonatal sepsis, including $12 \mathrm{~K}$. pneumoniae [17-24]. Together, these works from India report ST information for a single ESBL-producing strain (ST2318) and 24 CP strains; five each of ST231 and ST347; two each of ST29, ST147, ST1224 and ST2558; and one each of ST11, ST43 and ST101. Carbapenemase genes were reported for 21 of the CP strains and included 11 $b l a_{\mathrm{NDM}-1}, 8 b l a_{\mathrm{OXA}-48-\text { like, }}$ one each of $b l a_{\mathrm{NDM}-7}$ and $b l a_{\mathrm{KPC}}$. A single report from Nepal described two neonatal sepsis outbreaks [7], the first caused by ST15 carrying $b l a_{\mathrm{NDM}-1}$ and $b l a_{\mathrm{CTX}-\mathrm{M}-15}$, and the second by ST1559 carrying $b l a_{\mathrm{CTX}-\mathrm{M}-15}$ without any carbapenemases. The final report described $K$. pneumoniae BSI isolates from Hong Kong that produced DHA enzymes (resulting in third-generation cephalosporin resistance). Among the genotyped isolates were four ST11, and one each of ST17, ST23 and ST39 [25].

It is clear from these reports that several well-known globally distributed CP/ESBL-associated STs are present in S/SE Asia, e.g. STs 14, 15, 17, 29, 101, 147 and 231, a proposition that is further supported by studies exploring $K$. pneumoniae isolates from a broader range of clinical specimen types [12, 13, 15, 26, 27]. However, there remains a clear lack of systematic studies with which to fully understand the $\mathrm{CP}, \mathrm{ESBL}$ and broader population genotypes of $K$. pneumoniae causing BSI in these regions.

Capsule- or lipopolysaccharide- (LPS) targeted immunisation against $K$. pneumoniae has been proposed as an alternative strategy for prevention or therapy of MDR BSI. Hence, capsular serotype (K-type) and LPS (O-type) profiles among BSI isolates are also of interest. More than 130 capsular serotypes have been predicted on the basis of genome data [28]. Among the studies identified through the formal search described above, two did not report K-types and three included only a limited assessment, i.e. PCR screening for a small number of types that are associated with enhanced virulence (K1 and $\mathrm{K} 2$ $[17,24]$, or K1, K2, K5, K20, K54 and K57 [23]). Among the remaining three studies, two reported single K17 and K64 CP isolates in India [19, 20], while the Nepalese outbreak study reported the KL14 capsule locus, encoding capsule type K14 (ST1559) and a novel locus that was later defined as KL112 (ST15) [7]. None of these studies reported O-types. We searched PubMed for additional studies reporting $\mathrm{K}$ - and O-types of $\mathrm{K}$. pneumoniae BSI in our regions of interest (search terms in "Methods"), revealing one additional report describing 47 isolates (including 28 BSI isolates) from a suspected neonatal intensive care unit outbreak in Madras, India [29]: Thirty-eight isolates were shown to be closely related by pulsed-field gel electrophoresis (MLST not reported), all of which expressed the same novel $\mathrm{K}$ and $\mathrm{O}$ serotype. Among the remaining nine unrelated isolates, three K2:O1 were identified in addition to one each of K23:O1, K54:O1, K62:O1, K62:O2, K-non-typeable:O1 and K2:O-novel. 
The capsule, alongside the LPS, type I and type III fimbriae, and the enterobactin siderophore, are common to all $K$. pneumoniae strains and are required for pathogenesis. A number of accessory virulence determinants (including RmpA/RmpA2, which upregulate capsule expression; the colibactin genotoxin; and the yersiniabactin, aerobactin and salmochelin siderophores that promote systemic survival and dissemination [30-34]) are overrepresented among isolates causing invasive disease in human population studies [35-37]. Each has also been shown to enhance virulence in murine infection models through comparison of isogenic knockout mutant strains [31-34]. K. pneumoniae expressing several of these determinants are associated with severe community-acquired invasive disease, often manifesting as liver abscess. These are classified as "hypervirulent" infections; they occur globally but are most commonly reported in SE Asia [38]. They are predominantly associated with clones ST23, ST86 and ST65 [39, 40], which typically express the $\mathrm{K} 1 / \mathrm{K} 2$ capsules. The dominance of a small number of clones combined with strong linkage between the $\operatorname{rmp} A / A 2$, aerobactin and salmochelin synthesis loci (which are co-located on virulence plasmids $[41,42])$ has created difficulties for understanding the relative importance of these key virulence factors. However, recent studies implicate aerobactin as the most important siderophore and a key biomarker for hypervirulence alongside the peg-344 transporter gene (sometimes misannotated as pagO, in strong genetic linkage with aerobactin due to being located nearby on the same virulence plasmid) [37, 43, 44]. Notably, despite its apparent relevance as a hypervirulence biomarker, peg-344 isogenic knockout mutants did not show reduced virulence in either of two independent murine models of hypervirulent disease [44, 45].

To date the majority of hypervirulent $K$. pneumoniae have remained susceptible to antimicrobials [38, 46] (except ampicillin). However, the last few years have seen increasing reports of "convergent" $K$. pneumoniae that are both hypervirulent (defined as strains carrying the iuc aerobactin locus) and MDR ESBL/CP either due to acquisition of an MDR plasmid by a hypervirulent strain [47-51] or by acquisition of a virulence plasmid by an MDR strain [49, 52-56]. The majority of these reports represent sporadic isolations. However in 2017, it was reported that an outbreak of ventilator-associated pneumonia in a Chinese hospital, resulting in five deaths, was caused by a CP ST11 strain that had acquired a virulence plasmid harbouring the iuc locus, plus rmpA2 (without the salmochelin locus (iro), rmpA or peg-344) [52]. Notably, the outbreak strain showed enhanced virulence in the Galleria mellonella infection model compared to a wild-type ST11 lacking the virulence plasmid and a plasmid cured variant [52], further supporting the importance of the iuc locus. Subsequent retrospective investigations revealed that similar CP ST11 iuc + strains had already been silently spreading within China prior to the initial report $[52,57]$.

Given the high burden of both MDR and hypervirulent K. pneumoniae infections, S/SE Asia may represent a hub for MDR-virulence convergence, with the potential for outbreaks of severe disease with extremely limited treatment options such as that reported in China [52]. However, so far no studies have provided a complete molecular epidemiological picture (STs, serotypes, AMR and hypervirulence determinants) of $K$. pneumoniae BSI agents in S and SE Asia, leaving a gap in our knowledge about the local pathogen population and the prevalence and/or diversity of convergent strains. Here we present a genomic epidemiology study of BSI K. pneumoniae from seven major healthcare facilities across S/SE Asia, leveraging a recently established genomic framework that incorporates rapid genotyping of clinically important features (ST, AMR, hypervirulence, and capsule and lipopolysaccharide synthesis loci). The data are highly relevant to the design of $K$. pneumoniae control strategies, revealing a diverse population with high rates of AMR and virulence loci, and high prevalence of convergent strains.

\section{Methods \\ Literature review}

To identify studies reporting molecular data on $K$. pneumoniae BSI in our regions of interest, we searched PubMed with no language restrictions for reports that contained the terms "(Klebsiella)" and "((India*) OR (Nepal*) OR (Viet*) OR (Hong Kong) OR (Cambodia) OR (Lao*) OR (Thai*) OR (Asia))" and "(blood*) OR (sepsis) OR (bacteraemia) OR (bacteremia)" and "(MLST) OR (sequence type) OR (multi-locus sequence typ*) OR (genom*)". To check for other studies reporting $K$. pneumoniae serotypes in the region, we searched for "(Klebsiella)" and "((India*) OR (Nepal*) OR (Viet*) OR (Hong Kong) OR (Cambodia) OR (Lao*) OR (Thai*) OR (Asia))" and "(blood*) OR (sepsis) OR (bacteraemia) OR (bacteremia)" and "(serotyp") OR (capsul") OR (Ktype) OR (O-type) OR (LPS) OR (lipopolysaccharide)”.

\section{Setting}

The following tertiary care hospitals were included: Patan Hospital, Kathmandu, Nepal; Christian Medical College Hospital, Vellore, India; Angkor Hospital for Children, Siem Reap, Cambodia; Mahosot Hospital, Vientiane, Lao PDR; The Hospital of Tropical Diseases, Ho Chi Minh City, Vietnam; Prince of Wales Hospital, Hong Kong. Isolates were also provided from healthcare inpatient departments serviced by the Shoklo Malaria Research Unit, Mae Sot, Thailand. 


\section{Bacterial isolates and culture}

K. pneumoniae isolates obtained from blood cultures derived from hospital- and/or community-associated BSI, following routine diagnostic protocols in each hospital laboratory and identified using biochemical testing (typically API-20E, bioMerieux), were included in the study. Isolates available for sequencing represented the following fractions of $K$. pneumoniae BSI collected at each site in participating years: India, 10\%; Hong Kong, 17\%; Vietnam, 20\%; other sites, $>90 \%$ (Fig. 1). All sequenced isolates represent unique patient infection episodes.

\section{DNA extractions, library preparation and sequencing}

All isolates were cultured in LB broth at $37^{\circ} \mathrm{C}$ overnight before DNA extraction. Multiplexed Nextera XT libraries were sequenced on Illumina platforms, generating $150 \mathrm{bp}$ paired-end reads. Eight isolates were selected for additional long-read sequencing using the Nanopore MinION R9 device as previously described [58].

\section{Genome assembly and genotyping}

Illumina adapter sequences were removed and reads were quality trimmed using TrimGalore v0.4.4 (https://
github.com/FelixKrueger/TrimGalore). Subsequently, draft de novo assemblies were generated using SPAdes v3.10.1 [59] optimised with Unicycler v0.4.7 [60]. We excluded from further analysis nine low-quality genome assemblies outside the expected size range (5-6.5 Mbp). Chromosomal MLST, virulence locus ( $y b t$, iro, iuc, $r m p A, r m p A 2)$, and acquired resistance genes (excluding the core ampicillin resistance gene bla $a_{\mathrm{SHV}}$ and $o q x A B$ efflux genes) were typed using Kleborate v0.3.0 (https:// github.com/katholt/Kleborate). The peg-344 gene was identified using BLASTn search of the genome assemblies (query sequence accession BAH65947.1, $\geq 90 \%$ coverage and identity). Capsule (K) and lipopolysaccharide (O) loci were identified using Kaptive [28, 61]. Note that the KL1-KL77 loci are associated with the serologically defined capsule types K1-K77, respectively [62]. Serological types are yet to be resolved for the remaining loci (KL101-KL161), which were defined previously on the basis of gene content such that they are predicted to encode distinct capsule types [28,63]. Novel K-loci were manually extracted using Bandage [64] and annotated using Prokka v1.13.3 [65]. Where Kaptive was unable to confidently identify a K-locus due to fragmented genome
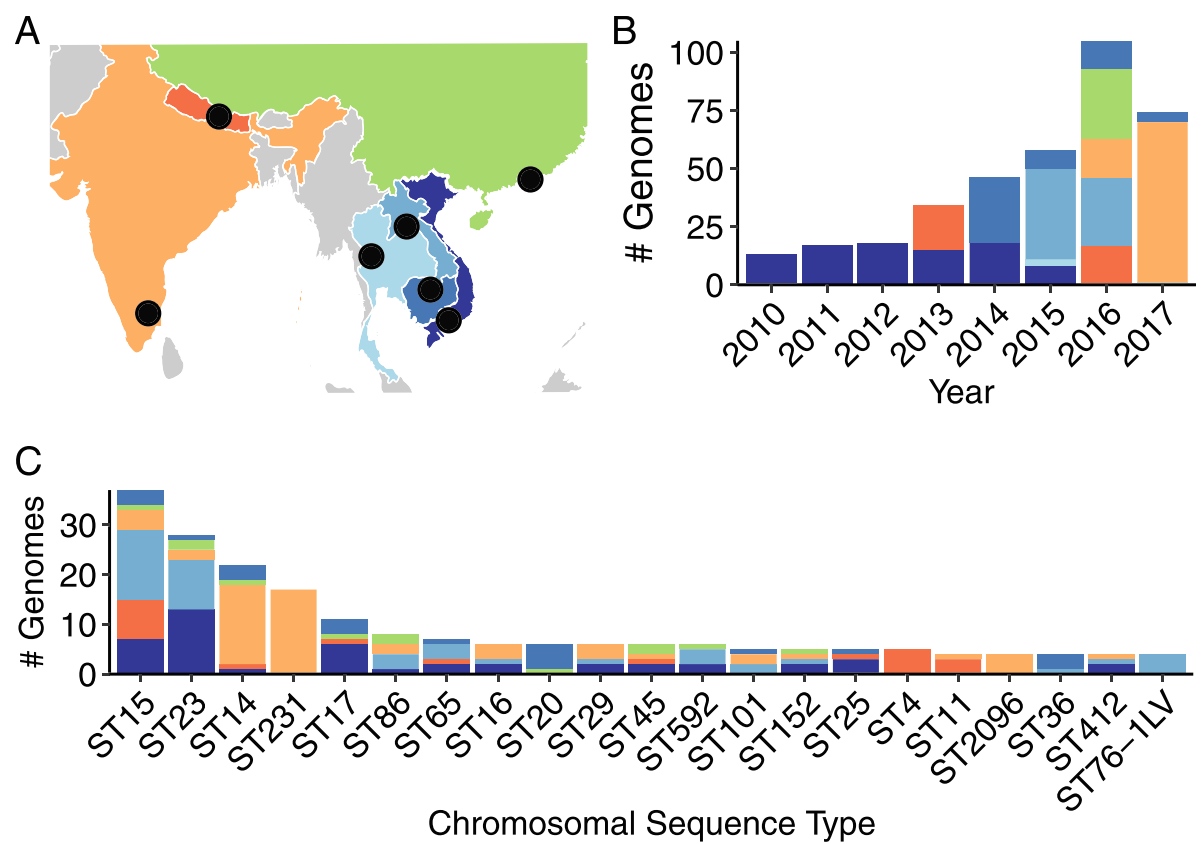

Location:

South Asia:

Southeast Asia:

Other:

India $\quad$ Nepal
Cambodia Laos Thailand $\square$ Vietnam
Hong Kong

Fig. 1 Klebsiella pneumoniae BSI isolates included in this study. a Collection sites and countries of origin for all Klebsiella pneumoniae complex isolates for which genome data were available. $\mathbf{b}$ Years of collection coloured by country of origin as in panel a. c Chromosomal multi-locus sequence types (STs) of Klebsiella pneumoniae sensu stricto isolates (only STs accounting for $>1 \%$ isolates are shown, coloured by country of origin as in panel $\mathbf{a})$ 
assemblies $(n=129)$, K-loci were predicted from $w z i$ alleles as described previously [66]. Wzi allelic typing was considered the gold-standard capsule genotyping method prior to the introduction of full-locus-based typing. However, when genome data are available, the latter approach is preferred because the K-locus is subject to chromosomal recombinations that can lead to a breakdown of associations between individual $w z i$ allelic variants and K-locus types [28]. As a result, a single $w z i$ allele may be associated with multiple distinct K-loci. Such alleles were identified among 29 genomes in this study, resulting in ambiguous K-locus calls that were excluded from further analyses. An additional 11 and one genomes harboured novel or missing $w z i$ alleles, respectively for which no K-locus predictions were possible.

Catpac [67] was used to calculate pairwise nucleotide differences between genomes after reassembly with SKESA v2.2.1 [68] (a highly conservative assembly approach, which reduces the likelihood of false-positive nucleotide differences). These data were used to identify clusters of isolates for which the genomes differed by $\leq$ 100 and $\leq 25$ single nucleotide variants (SNVs). Isolates from the same facility and separated by $\leq 25$ SNVs can be considered putative nosocomial transmission clusters [67, 69, 70]; distances $\leq 100$ SNVs also imply descent from a recent common ancestor, but are more consistent with community or between-facility transmission, or regional spread between countries [70].

Hybrid Illumina-Nanopore assemblies were generated using Unicycler v0.4.7 as described previously [58]. Assemblies were annotated using Prokka v1.13.3 and $i u c+$ plasmid annotations were manually curated before depositing in GenBank (accessions listed in Table 4). Plasmid replicon types were identified using the PlasmidFinder database v2.0 [71]. Isolate information, genotypes and genome data accessions are provided in Additional file 1: Table S1. Genome assemblies, novel plasmid and K-locus sequences are also available in Figshare [72]: https://doi.org/10.26180/5c67982956721.

\section{Statistical analyses}

Statistical analyses were performed in R v3.3.3 [73], and data were visualised using ggplot2 v2.2.1 [74]. Given the disparity in sampling frames and small subgroup sample sizes, it was not appropriate to test trends at a country or year level. Statistical tests for regional differences in genetic features between $K$. pneumoniae populations (Table 1) were calculated using the subset of $K$. pneumoniae collected in overlapping 2-year periods: S Asia region (Nepal and India; 2016-2017; $n=102$ ) vs SE Asia region (Cambodia, Laos, Thailand, Vietnam; 2015-2016, $n=100$ ).

\section{Results}

The genomes of 393 presumptive K. pneumoniae BSI from seven countries across S/SE Asia were sequenced
(Fig. 1a, Additional file 1: Table S1). Twenty-eight genomes were identified as Enterobacteriaceae species outside of the $K$. pneumoniae species complex and were excluded. Among the remaining 365 isolates, the majority $(n=331,91 \%)$ of organisms were confirmed to be $K$. pneumoniae. Among the regional comparator samples, these accounted for a higher proportion of genomes from S than SE Asia (98\% vs $87 \%, \quad p=0.011$; Table 1). The remaining isolates (Additional file 2: Table S2) were $K$. quasipneumoniae subsp. similipneumoniae $(n=20,5.5 \%), K$ variicola $(n=9$, $2.5 \%)$ and $K$. quasipneumoniae subsp. quasipneumoniae $(n=5,1.4 \%)$, which are indistinguishable from K. pneumoniae by microbiological methods $[35,67]$. Unfortunately, we did not have access to patient clinical data and hence were not able to assess the likelihood that individual BSI cases were hospital- or community-acquired, or to explore patient co-morbidities, which we expect to be quite diverse.

The $331 \mathrm{~K}$. pneumoniae were highly diverse and comprised 120 individual STs (Simpson's diversity index = $0.97)$, the majority (61\%) of which were represented by a single isolate. Nevertheless, we observed four common STs that each accounted for $>5 \%$ of the sequenced organisms: ST15 ( $n=37,11 \%)$, ST23 $(n=28,8.5 \%)$, ST14 $(n=22,6.6 \%)$ and ST231 ( $n=17,5 \cdot 1 \%$; Fig. 1c). Among these, only ST15 was common across all sites, whereas ST23 was significantly associated with SE Asia ( $p=$ $0.015)$ and ST14 and ST231 were both significantly associated with S Asia $(p<0.01$; Table 1$)$.

\section{Predicted capsular $(\mathrm{K})$ and $\mathrm{O}$ antigen serotypes}

In this collection of invasive $K$. pneumoniae from BSI, we detected 63 different K-loci including four novel loci designated KL162-165 (GenBank accessions: MK593451MK593454, Simpson's diversity index $=0.95$ ), the majority (67\%) of these K-loci were found in $\leq 3 \mathrm{~K}$. pneumoniae isolates each (Additional file 2: Table S3). The most common K-loci were KL1 ( $n=31$; including 28 ST23), KL2 $(n=27$; numerous STs including ST14, ST25, ST65 and ST86), KL51 ( $n=23$; including 17 ST231) and KL24 $(n=20$; including 17 ST15), together accounting for $28 \%$ of $K$. pneumoniae BSI (Fig. 2). Saliently, while ST23 and ST231 were each associated with only a single K-locus (KL1 and KL51, respectively), the two other most common STs were each associated with multiple K-loci (ST15: KL24=17, KL112 = 9, $\mathrm{KL} 10=3, \mathrm{KL} 62=2, \mathrm{KL} 19=2 ; \mathrm{ST} 14: \mathrm{KL} 64=7, \mathrm{KL} 2=6$, KL157 =1), as has been previously observed for ST258 [61]. Ten of the 12 previously described $\mathrm{O}$ antigen encoding loci were also detected (Simpson's diversity index $=0 \cdot 80$ ). Loci predicted to encode serotypes $\mathrm{O} 1$ and $\mathrm{O} 2$ were the most common, together accounting for $71 \%$ of $K$. pneumoniae BSI (Fig. 2, Additional file 2: Table S4). Notably, the O3b locus, which is considered to be rare [63], was detected here at $8 \%$ prevalence across sites (mean $7 \%$ per site, range $0-11 \%$ ). 
Table 1 Comparison of key features of Klebsiella genomes from South and Southeast Asian sites

\begin{tabular}{|c|c|c|c|c|c|c|c|}
\hline & S Asia & SE Asia & $p$ value (adj) & OR & $\mathrm{LCl}$ & $\mathrm{UCl}$ & \\
\hline Species assignment & 102 & 100 & & & & & \\
\hline K. pneumoniae & $100(98 \%)$ & $87(87 \%)$ & 0.011 & 0.13 & 0.01 & 0.62 & * \\
\hline K. variicola & $1(1 \%)$ & $4(4 \%)$ & 0.838 & 4.18 & 0.40 & 209.04 & \\
\hline K. quasipneumoniae ssp. quasipneumoniae & $1(1 \%)$ & $0(0 \%)$ & na & na & na & na & \\
\hline K. quasipneumoniae ssp. similipneumoniae & $0(0 \%)$ & $9(9 \%)$ & na & na & na & na & \\
\hline \multicolumn{8}{|l|}{ Features of $K$. pneumoniae sensu stricto } \\
\hline Sequence type assignment & 100 & 87 & & & & & \\
\hline ST15 & $11(11 \%)$ & $15(17 \%)$ & 1.158 & 1.68 & 0.67 & 4.32 & \\
\hline ST14 & $16(16 \%)$ & $2(2 \%)$ & 0.008 & 0.12 & 0.01 & 0.56 & ** \\
\hline ST23 & $2(2 \%)$ & $12(14 \%)$ & 0.015 & 7.76 & 1.65 & 73.39 & * \\
\hline ST231 & $17(17 \%)$ & $0(0 \%)$ & $5.14 \times 10^{-5}$ & 0.00 & 0.0 & 0.24 & ** \\
\hline Capsular serotype prediction & 79 & 87 & & & & & \\
\hline KL1 & $2(2.5 \%)$ & $13(15 \%)$ & 0.012 & 6.70 & 1.44 & 63.22 & * \\
\hline KL2 & $4(5.1 \%)$ & $11(13 \%)$ & 0.216 & 2.70 & 0.76 & 12.15 & \\
\hline Antimicrobial resistance prediction & 100 & 87 & & & & & \\
\hline Multi-drug resistant ( $\geq 3$ acquired classes) & $81(81 \%)$ & $44(51 \%)$ & $1.06 \times 10^{-4}$ & 0.24 & 0.12 & 0.48 & ** \\
\hline Aminoglycosides & $76(76 \%)$ & $41(47 \%)$ & 0.001 & 0.28 & 0.14 & 0.55 & $* *$ \\
\hline Fluoroquinolones & $80(80 \%)$ & $39(45 \%)$ & $7.13 \times 10^{-6}$ & 0.21 & 0.10 & 0.41 & $* *$ \\
\hline Phenicols & $27(27 \%)$ & $25(29 \%)$ & 7.833 & 1.09 & 0.55 & 2.17 & \\
\hline Sulfonamides & $63(63 \%)$ & $42(48 \%)$ & 0.493 & 0.55 & 0.29 & 1.02 & \\
\hline Tetracyclines & $24(24 \%)$ & $39(45 \%)$ & 0.029 & 2.56 & 1.32 & 5.05 & * \\
\hline Trimethoprim & $62(62 \%)$ & $38(44 \%)$ & 0.118 & 0.48 & 0.25 & 0.89 & \\
\hline 3rd-generation cephalosporins (ESBL) & $60(60 \%)$ & $41(47 \%)$ & 0.949 & 0.60 & 0.32 & 1.11 & \\
\hline Carbapenems & $47(47 \%)$ & $1(1 \%)$ & $7.13 \times 10^{-13}$ & 0.01 & 0.0 & 0.08 & ** \\
\hline Virulence prediction & 100 & 87 & & & & & \\
\hline Yersiniabactin $(y b t)$ & $65(65 \%)$ & $46(53 \%)$ & 0.205 & 0.61 & 0.32 & 1.13 & \\
\hline Aerobactin (iuc) & $27(27 \%)$ & $32(37 \%)$ & 0.319 & 1.57 & 0.81 & 3.07 & \\
\hline luc lineages & 100 & 87 & & & & & \\
\hline inc 1 & $13(13 \%)$ & $27(31 \%)$ & 0.012 & 2.99 & 1.36 & 6.86 & * \\
\hline iuc 3 & $0(0 \%)$ & $5(6 \%)$ & 0.061 & na & 1.08 & $\inf$ & \\
\hline iuc 5 & $12(12 \%)$ & $0(0 \%)$ & 0.001 & 0.00 & 0.0 & 0.8 & $* *$ \\
\hline$A M R$ and virulence convergence & 100 & 87 & & & & & \\
\hline $\mathrm{ESBL}$ and $y b t$ & $45(45 \%)$ & $26(30 \%)$ & 0.144 & 0.52 & 0.27 & 0.99 & \\
\hline ESBL and iuc & $16(16 \%)$ & $1(1 \%)$ & 0.001 & 0.06 & 0.0 & 0.41 & $* *$ \\
\hline $\mathrm{CP}$ and $y b t$ & $37(37 \%)$ & $1(1 \%)$ & $1.79 \times 10^{-10}$ & 0.02 & 0.0 & 0.13 & ** \\
\hline $\mathrm{CP}$ and $i \mathrm{CC}$ & 13 (13\%) & $0(0 \%)$ & na & na & 0.0 & 0.34 & \\
\hline
\end{tabular}

*South (S) Asia is represented by the two sites in India and Nepal, isolated 2016-2017; Southeast (SE) Asia is represented by the sites in Cambodia, Laos, Thailand and Vietnam, isolated 2015-2016. $p$ values were calculated using Fisher's exact test for differences in the prevalence of each feature (in rows) for the S vs SE Asia samples and are adjusted using Bonferroni correction for the number of tests within each group of comparisons (as labelled in italics; sample size for each are also in italics). na test not applicable (one or more values equal to zero). Inf infinity, OR odds ratio, $\mathrm{LCl}$ and $\mathrm{UCl}$, lower and upper bounds for $95 \%$ confidence interval, respectively. ${ }^{*} p<0.05,{ }^{* *} p<0.01$

\section{Acquired antimicrobial resistance determinants}

Acquired AMR determinants were detected in 91\% of $K$. pneumoniae genomes. The number of antimicrobial classes to which each isolate was predicted to be resistant showed a bimodal distribution (Fig. 3a), with the majority of $K$. pneumoniae being MDR (acquired AMR genes conferring resistance to $\geq 3$ drug classes; $63 \%$ ) or possessing no acquired AMR genes (9\%). The prevalence of MDR differed between sampling locations and ranged from $22 \%$ in Hong Kong to $85 \%$ in India. MDR was 

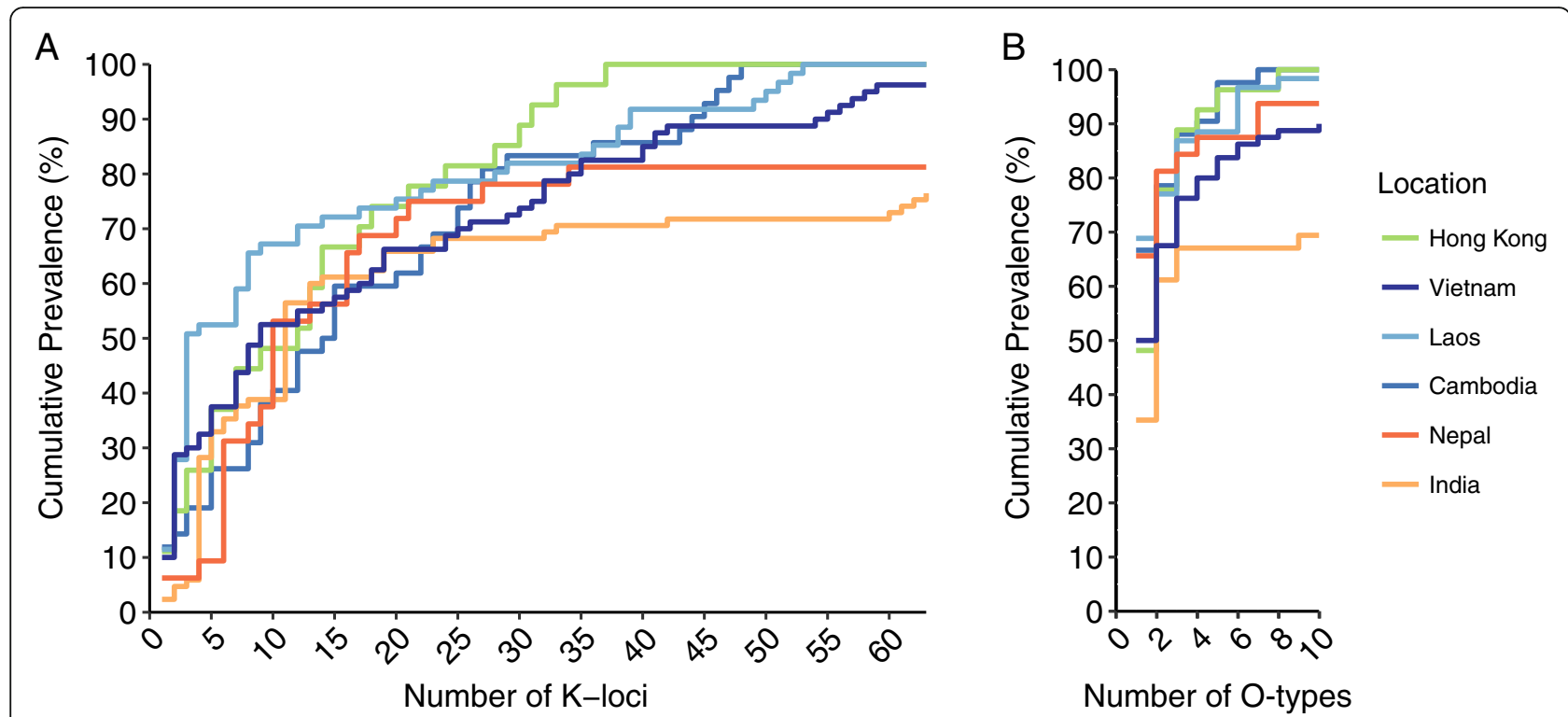

Fig. 2 Cumulative $\mathrm{K}$ and $\mathrm{O}$ locus prevalence by location. a Cumulative prevalence of K-loci ordered by mean prevalence across all locations (highest to lowest, see Additional file 2: Table S3). b Cumulative prevalence of predicted O-types ordered by mean prevalence across all locations (highest to lowest, see Additional file 2: Table S4). Note that the Thai sampling site is excluded due to small sample size $(n=4)$

significantly more prevalent among $\mathrm{S}$ Asian isolates than SE Asian isolates ( $81 \%$ vs $51 \%, p=0.0001$; Table 1$)$. Consistently, the $\mathrm{S}$ Asian organisms had a significantly higher prevalence of fluoroquinolone, aminoglycoside and carbapenem resistance determinants than the SE Asian organisms $(p<0.001$ for each class; Table 1).

The overall prevalence of ESBL genes among the $K$. pneumoniae isolates was $47 \%(n=157)$, but varied between study sites (22-75\%; Fig. 3b). The majority of ESBL K. pneumoniae (90\%) were predicted to be resistant to a further $\geq 3$ alternative antimicrobial classes (median 7 classes). The most common ESBL genes were bla $_{\mathrm{CTX}-\mathrm{M}-15}(n=120 / 157,76 \%), b_{\text {CTX-M-27 }}(n=14 / 157$,
9\%) and bla $_{\mathrm{CTX-M-14}}(n=13 / 157,8 \%)$; these were detected in diverse chromosomal STs (Table 2, Additional file 1: Table S1). Twelve additional putative ESBL genes were also detected in 1-7 genomes each (Additional file 1: Table S1).

The overall prevalence of carbapenemase genes was $17 \%(n=57)$, again varying widely between sites $(0-50 \%$; Fig. 3c). All isolates with a carbapenemase gene were also MDR, with predicted resistance to a median of six drug classes. The most common carbapenemases were the OXA-48-like bla $a_{\text {OXA-232 }}(n=36 / 57 ; 63 \%)$ and the metallo-beta-lactamase bla $_{\mathrm{NDM}-1} \quad(n=18 / 57 ; 32 \%$, including four genomes with $\left.b l a_{\mathrm{OXA}-232}\right)$; again, these were
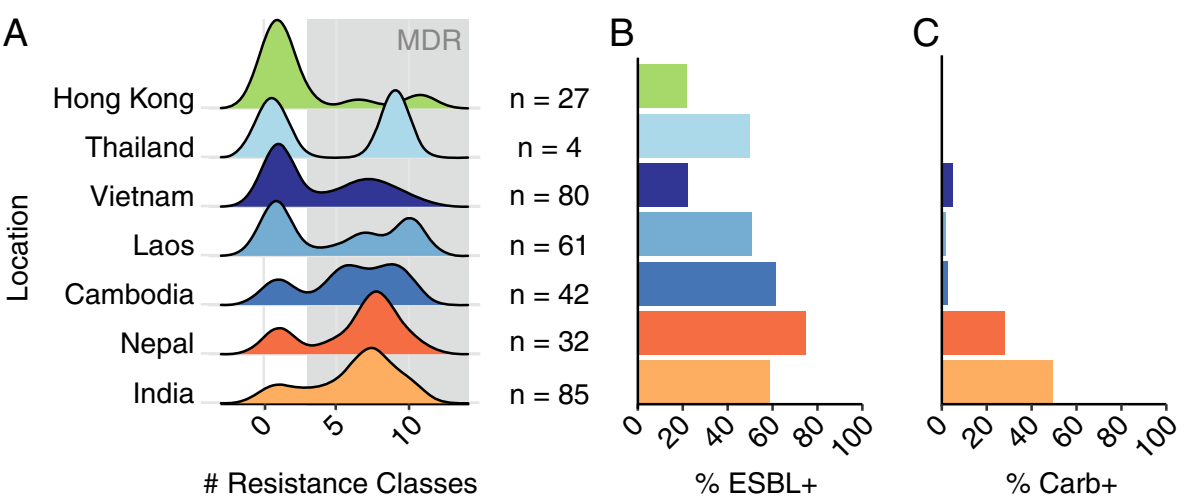

Fig. 3 Prevalence of antimicrobial resistance determinants among K. pneumoniae sensu stricto isolates. a Density plots showing the distributions of number of drug classes for which acquired resistance determinants were detected in each genome ( $n=$ total genomes by location). Grey shading indicates multi-drug resistance ( $\geq 3$ resistance classes). b Proportion of genomes for which extended-spectrum beta-lactamase genes were detected (\% ESBL+). c Proportion of genomes for which carbapenemase genes were detected (\% Carb+) 
Table 2 Notable AMR STs by location

\begin{tabular}{|c|c|c|c|c|c|c|c|c|}
\hline Location & Total ESBL (\%) & Total CP (\%) & Notable clone/s & $N$ & N ESBL (\% of ESBL/site) & ESBL genes $(N)$ & N CP (\% of CP/site) & $C P$ genes $(N)$ \\
\hline Hong Kong & $5(19 \%)$ & 0 & - & - & - & - & - & - \\
\hline Vietnam & $20(25 \%)$ & $4(5 \%)$ & * ST15 & 7 & $4(20 \%)$ & $\begin{array}{l}\text { CTX-M-15 (3) } \\
\text { CTX-M-14 (2) }\end{array}$ & $2(50 \%)$ & NDM-1 (1) \\
\hline Thailand & $2(50 \%)$ & 0 & - & - & - & - & - & - \\
\hline \multirow[t]{2}{*}{ Laos } & $32(53 \%)$ & $1(2 \%)$ & * ST15 & 14 & $14(44 \%)$ & CTX-M-15 (14) & 0 & - \\
\hline & & & ST76-1LV & 4 & $4(13 \%)$ & CTX-M-15 (4) & 0 & - \\
\hline \multirow[t]{4}{*}{ Cambodia } & 27 (64\%) & $1(2 \%)$ & ST20 & 5 & $5(19 \%)$ & $\begin{array}{l}\text { CTX-M-27 (3) } \\
\text { CTX-M-14 (2) } \\
\text { CTX-M-15 (1) }\end{array}$ & 0 & - \\
\hline & & & * ST15 & 3 & $3(11 \%)$ & $\begin{array}{l}\text { CTX-M-15 (3) } \\
\text { CTX-M-27 (1) }\end{array}$ & 0 & - \\
\hline & & & ST36 & 3 & $3(11 \%)$ & CTX-M-15 (2) & 0 & - \\
\hline & & & * ST15 & 8 & $8(33 \%)$ & CTX-M-15 (8) & $6(67 \%)$ & NDM-1 (5) \\
\hline \multirow[t]{2}{*}{ Nepal } & $24(75 \%)$ & $9(28 \%)$ & ST4 & 5 & $5(21 \%)$ & CTX-M-15 (5) & 0 & - \\
\hline & & & ST11 & 3 & $3(13 \%)$ & SFO-1 (3) & $3(33 \%)$ & OXA-232 (3) \\
\hline \multirow[t]{6}{*}{ India } & 52 (61\%) & $42(49 \%)$ & ST231 & 17 & $13(25 \%)$ & CTX-M-15 (13) & $13(31 \%)$ & OXA-232 (13) \\
\hline & & & ST14 & 16 & $7(13 \%)$ & CTX-M-15 (7) & $11(26 \%)$ & OXA-232 (10) \\
\hline & & & * ST15 & 4 & $3(6 \%)$ & CTX-M-15 (3) & 0 & - \\
\hline & & & ST16 & 3 & $3(6 \%)$ & CTX-M-15 (3) & $2(5 \%)$ & NDM-1 (1) \\
\hline & & & ST29 & 3 & $3(6 \%)$ & CTX-M-15 (3) & 0 & - \\
\hline & & & ST395 & 3 & $1(25 \%)$ & CTX-M-15 (1) & $3(7 \%)$ & OXA-232 (3) \\
\hline
\end{tabular}

Total ESBL and Total CP: total number of genomes carrying ESBL and carbapenemase genes per site, respectively

Notable clones: clones with $\geq 3 \mathrm{ESBL}$ and/or carbapenemase gene-positive genomes at any single site. ${ }^{*} \mathrm{ST} 15$ is the only clone meeting these criteria at $>1$ site

$N$ : total number of genomes of each notable clone at each site (includes all genomes assigned to the ST regardless of predicted AMR)

$N$ ESBL: number of genomes of each notable clone with at least one ESBL and no carbapenemase genes per site

ESBL genes: details of the ESBL genes detected in each notable clone at each site

$N C P$ : number of genomes of each notable clone with at least one carbapenemase gene per site

$\mathrm{CP}$ genes: details of the carbapenemase genes detected in each notable clone at each site

each detected in a diverse set of STs (Table 2, Additional file 1: Table S1). Five other carbapenemase genes were detected in 1-4 genomes each (Additional file 1: Table S1); bla $a_{\mathrm{KPC}}$ was not detected. Details of ESBL/CP K. pneumoniae STs identified at each site, including their specific enzymes, are shown in Table 2. Notably ST15 carrying bla ${ }_{\text {СTX-M-15 }}$ were identified at all sites except Thailand, and occasionally also harboured the carbapenemases $b l a_{\mathrm{NDM}-1}$ or $b l a_{\mathrm{OXA}-232}$.

\section{Acquired virulence determinants}

We identified genes linked to invasive disease: the yersiniabactin locus $(y b t)$ was present in 163 (49\%) of the BSI K. pneumoniae, with the site prevalence ranging from 19 to $67 \%$ (Fig. 4a); no significant difference in $y b t$ prevalence was observed between $\mathrm{S}$ and SE Asia (Table 1). Nine of the 14 known chromosomally integrated $y b t$ mobile-genetic elements (ICEKps [36, 75, 76]) and two $y b t$ plasmids were detected. The most common were ICEKp5 $(n=41), \operatorname{ICEKp} 4(n=36)$ and ICE $K p 10$ (also encoding colibactin, $n=31$ ), found across multiple study sites. The ICEKp10-positive samples included 25 in clonal group 23 (ST23 plus related STs), wherein
ICEKp 10 is a marker of the important globally distributed CG23-I sub-lineage [77].

The iuc, iro, rmpA, rmpA2 and peg-344 loci, typically carried on virulence plasmids, were commonly detected in our genome collection (28\% iuc, $21 \%$ iro, $18 \% \mathrm{rmpA}$ 16\% rmpA2, 19\% peg-344; 9\% with all five). Peg-344 and iuc have been suggested as the most predictive for hypervirulence [37]. We focus the remainder of our analyses on the iuc locus because its mechanism of action is well understood and its isogenic knockout mutants are clearly attenuated in mouse models of hypervirulent infection, unlike those for peg-344 [32, 43-45].

The prevalence of iuc did not differ significantly between the S and SE Asian isolates, but iuc lineages and chromosomal STs of iuc-positive isolates were differentially distributed between the sampling sites (Fig. 4b, c, Tables 1 and 3). Iuc lineage 1 (iuc1, $n=66$ ) was widely distributed (Fig. 4c) but more prevalent in SE Asia (27\% vs $13 \%$ in S Asia, $p=0.011$; Table 1). Iuc1 is associated with the KpVP-1 virulence plasmid [78] and was detected among $15 \mathrm{~K}$. pneumoniae STs, most commonly those known to be associated with hypervirulent infections: ST23 $(n=28)$, ST65 $(n=7)$ and ST86 $(n=7)$. Iuc5 


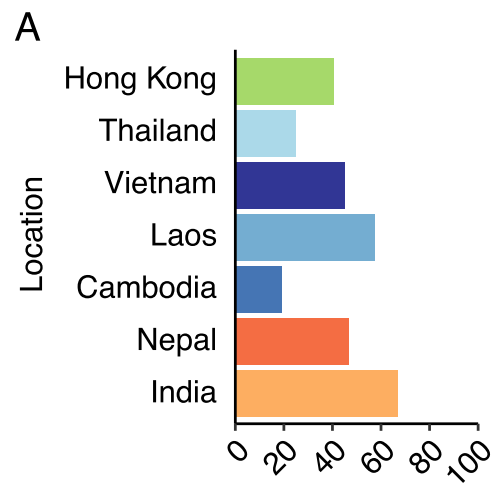

$\% y b t+$

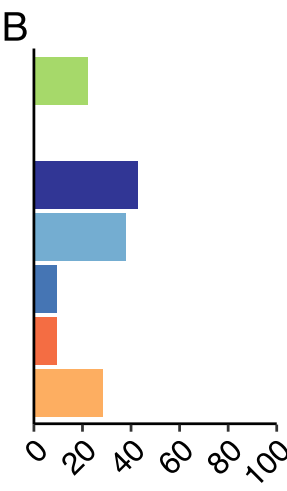

$\%$ iuc +

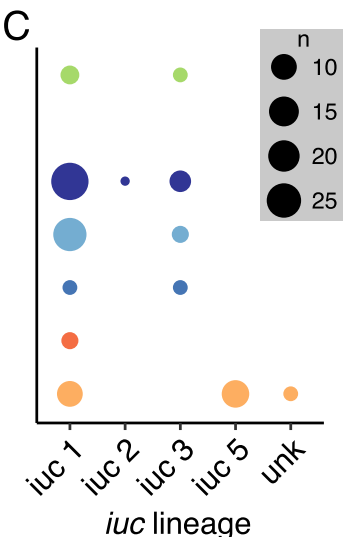

iuc lineage

Fig. 4 Prevalence of key virulence determinants among K. pneumoniae sensu stricto isolates. a Proportion of genomes for which the yersiniabactin locus was detected (\% ybt+) by location. b Proportion of genomes for which the aerobactin locus was detected (\% iuct) by location. c iuc lineages by location. Points are scaled by the number of genomes as per legend. Unk; unknown

Table 3 Notable iuc-positive STs by region

\begin{tabular}{|c|c|c|c|c|c|c|c|c|}
\hline Location & Total iuct & Notable clone/s & iuc allele & N iuc (\% of iuc/site) & ICEKp (N) & iro allele $(N)$ & $r m p A / 2(N)$ & $\mathrm{ESBL} / \mathrm{CP}(\mathrm{N})$ \\
\hline Hong Kong & $6(22 \%)$ & ST45 & inc3 & $1(17 \%)$ & ICEKp4 (1) & - & - & CTX-M-3 (1) \\
\hline \multirow[t]{4}{*}{ Vietnam } & \multirow[t]{4}{*}{$34(43 \%)$} & * ST23 & incl & $13(38 \%)$ & $\begin{array}{l}\text { ICEKp10 } 0^{\mathrm{a}}(10) \\
\text { ICEKp3 (1) }\end{array}$ & irol (13) & (10) & CTX-M-14 (1) \\
\hline & & \multirow[t]{2}{*}{ * ST25 } & inc3 & $2(6 \%)$ & ICEKp1 (2) & iro3 (2) & $(2)$ & - \\
\hline & & & incl & $1(3 \%)$ & - & irol (1) & (1) & - \\
\hline & & * ST65 & incl & $2(6 \%)$ & ICEKp10 a (1) & irol (2) & (2) & CTX-M-15, VEB-1 (1) \\
\hline Thailand & 0 & - & & - & - & - & - & - \\
\hline \multirow[t]{4}{*}{ Laos } & \multirow[t]{4}{*}{$23(38 \%)$} & * ST23 & incl & $10(44 \%)$ & ICEKp10 a (10) & irol (10) & $(10)$ & CTX-M-63 (1) \\
\hline & & * ST65 & incl & $2(6 \%)$ & ICEKp10 a (2) & irol (3) & (3) & - \\
\hline & & * ST86 & incl & - & ICEKp4 (1) & irol (3) & (3) & - \\
\hline & & ST592 & incl & $10(44 \%)$ & - & irol (3) & (3) & - \\
\hline Cambodia & $4(10 \%)$ & - & - & - & - & - & - & - \\
\hline Nepal & $3(9 \%)$ & ST15 & incl & $2(67 \%)$ & ICEKp12 (2) & - & $(2)$ & $\begin{array}{l}\text { CTX-M-15 (2) } \\
\text { OXA-232 (1) }\end{array}$ \\
\hline \multirow[t]{5}{*}{ India } & \multirow[t]{5}{*}{$24(28 \%)$} & \multirow[t]{2}{*}{ ST231 } & iuc5 & $12(50 \%)$ & ICEKp5 (12) & - & - & $\begin{array}{l}\text { CTX-M-15 (8) } \\
\text { OXA-232 (8) }\end{array}$ \\
\hline & & & iuc ukn & $2(8 \%)$ & ICEKp5 (2) & - & - & $\begin{array}{l}\text { CTX-M-15 (2) } \\
\text { OXA-232 (2) }\end{array}$ \\
\hline & & ST2096 & incl & $3(13 \%)$ & ICEKp5 (2) & irol (1) & - & CTX-M-15 (2) \\
\hline & & * ST23 & incl & $2(8 \%)$ & $\begin{array}{l}\text { ICEKp3 (1) } \\
\text { ICEKp10 }{ }^{a}(1)\end{array}$ & irol (2) & (1) & CTX-M-15 (1) \\
\hline & & ST11 & iucl & $1(48 \%)$ & ICEKp12 (1) & - & - & $\begin{array}{l}\text { CTX-M-15, } \\
\text { OXA-232 (1) }\end{array}$ \\
\hline
\end{tabular}

Total iuc+: total number of genomes carrying iuc per site (i.e. predicted as aerobactin-producing)

Notable clones: clones with $\geq 3$ iuc-positive genomes at any single site, or carrying iuc in addition to ESBL and/or carbapenemase genes. *Known

hypervirulent STs

iuc allele: iuc lineages predicted by Kleborate. unk = iuc lineage unknown

$N$ iuc: number of genomes of each notable clone carrying iuc per site

ICEKp: ICEKp variants and the number of genomes of notable clones carrying these variants per site. ${ }^{\mathrm{a}} \mathrm{ICEKp} 10 \mathrm{carries}$ ybt and $c l b$

iro allele: iro lineage and the number of genomes of notable clones carrying these iro variants per site

$r m p A / 2$ : number of genomes of notable clones carrying the $r m p A$ and/or $r m p A 2$ loci per site

ESBL/CP: ESBL and carbapenemase genes detected among notable clones and the number of genomes carrying these genes per site 
( $n=12)$ is associated with $E$. coli plasmids and was detected only in ST231 from India, while iuc2 (associated with KpVP-2 [78]) was detected in a single ST380 isolate from Vietnam. Iuc3 $(n=13)$ was detected in SE Asia (Cambodia, Vietnam, Laos) and Hong Kong (Fig. 4c), among eight distinct STs (Additional file 1: Table S1). We selected four iuc3-positive isolates from Vietnam and Laos for long-read sequencing and found each harboured a distinct and novel $\mathrm{FIB}_{\mathrm{K}} / \mathrm{FII}$ iuc3 plasmid (Table 4).

\section{Genotypic convergence of antimicrobial resistance and virulence}

While strains carrying either AMR or hypervirulence determinants are of concern, those carrying both pose the greatest potential public health threat. The $y b t$ virulence locus was significantly associated with ESBL $K$. pneumoniae (OR 1.6; 95\% CI 1.06-2.55, $p=0.021$ ) and CP $K$. pneumoniae (OR3.5; 95\% CI 1.87-6.98, $p<0.0001$ ). $Y b t+\mathrm{ESBL} K$. pneumoniae BSI were detected at all sites (Fig. 5a) and exhibited a similar prevalence in both $\mathrm{S}$ and SE Asia (Table 1). Iuc was present in $13 \%$ of ESBL K. pneumoniae BSI (vs $43 \%$ among non-ESBL; OR 0.2;
95\% CI $0.12-0.38, p<0.0001)$ and $23 \%$ of CP $К$. pneumoniae (vs 30\% among non-CP; OR 0.8; 95\% CI $0.37-$ $1.59, p=0 \cdot 6$ ). Overall prevalence of $i u c+$ ESBL $K$. pneumoniae was $6 \%$. Iuc + ESBL isolates were detected at five of the seven sampling locations (Fig. 5a) and were more common in S than SE Asia $(p<0.001$, Table 1). Conversely, iuc + CP K. pneumoniae BSI were detected only in India ( $n=12$ ST231, $14 \%$ of Indian isolates) and Nepal $(n=1 \mathrm{ST} 15,3 \%)$.

Convergent AMR-hypervirulent isolates (organisms carrying iuc plus ESBL and/or carbapenemase genes) were seen across seven different STs circulating across this region, with a prevalence of $7.3 \%$ (Fig. 5b, Table 3). Long-read sequencing of four representative isolates of different STs revealed four novel mosaic plasmids. Three of these plasmids (and the four iuc3 plasmids) harboured iuc plus AMR genes (1-10 AMR genes, encoding resistance to 10 drug classes; Table 4). Notably, one of these plasmids harboured iuc1, bla $a_{\mathrm{CTX}-\mathrm{M}-15}$, and eight additional AMR genes (plasmid pBA813_1 from isolate BA813, Table 4). While pBA813_1 was not predicted to encode the tra plasmid transfer machinery, the four iuc3 plasmids and one of the iuc1 plasmids (pBA6201_1, iuc1

Table 4 Novel virulence plasmids sequenced in this study and their host isolate properties

\begin{tabular}{|c|c|c|c|c|}
\hline Isolate & Virulence plasmid (accession) & iuc & AMR genes & AMR genes elsewhere in genome \\
\hline $\begin{array}{l}\text { Sample data and } \\
\text { chromosomal } \\
\text { typing }\end{array}$ & Size and rep type(s) & lineage & & \\
\hline $\begin{array}{l}2579 \\
\text { Nepal, } 2016 \\
\text { ST15, KL112, ICEKP12 }\end{array}$ & $\begin{array}{l}\text { p2579_1 } \\
\text { (MK649822) } \\
\text { 182,805 bp } \\
\text { IncHI1B }\end{array}$ & iucl & - & 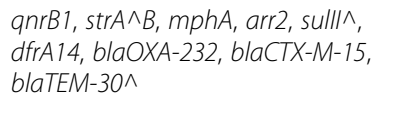 \\
\hline $\begin{array}{l}\text { BA6740 } \\
\text { India, } 2016 \\
\text { ST11, KL24, ICEKp12 }\end{array}$ & $\begin{array}{l}\text { pBA6740_1 (MK649823) } \\
226,590 \text { bp } \\
\text { IncFIB(pQIL), IncFIIK }\end{array}$ & lucl & qnrB1 & $\begin{array}{l}\text { strA } \wedge \text { B, rmtF, mphA, arr2, sulll, } \\
\text { blaOXA-232, blaCTX-M-15, blaSHV-11, } \\
\text { blaTEM-30^ }\end{array}$ \\
\hline $\begin{array}{l}\text { BA813 } \\
\text { India, } 2017 \\
\text { ST2096, KL64, ICEKp5 }\end{array}$ & $\begin{array}{l}\text { pBA813_1 } \\
\text { (MK649825) } \\
273,676 \text { bp } \\
\text { IncFIB(Mar), IncFIB }\end{array}$ & iucl & $\begin{array}{l}\text { blaCTX-M-15, blaOXA-232, } \\
\text { blaTEM-54^, aadA2^, armA, } \\
\text { msrE, mphE^, catA1^, sull, } \\
\text { tetD, dfrA12, dfrA14 }\end{array}$ & Sat- $2 A$, sull, dfra1, blaOXA-1 \\
\hline $\begin{array}{l}\text { BA6201 } \\
\text { India, } 2017 \\
\text { ST231, KL51, ICEKp5 }\end{array}$ & $\begin{array}{l}\text { pBA6201_1 (MK649824) } \\
\text { 195,016 bp } \\
\text { IncFIA, IncFIB(pQIL), IncFIIK, IncFII }\end{array}$ & inc 1 & $\begin{array}{l}r m t F, m p h A, e r m B \wedge, \operatorname{cat} A 1 \wedge \text {, } \\
\text { arr2 }\end{array}$ & blaCTX-M-15, blaTEM-30^ \\
\hline $\begin{array}{l}16114547 \\
\text { Laos, } 2016 \\
\text { ST290, KL21 }\end{array}$ & $\begin{array}{l}\text { p16114547_1 (MK649829) } \\
\text { 187,989 bp } \\
\text { IncFIBK, IncFII }\end{array}$ & inc 3 & anrS1, tetA^, blaTEM-30^ & blaSHV-26^ \\
\hline $\begin{array}{l}1675474 \\
\text { Laos, } 2015 \\
\text { ST7-1LV, KL54 }\end{array}$ & $\begin{array}{l}\text { p1675474_1 (MK649827) } \\
\text { 181,647 bp } \\
\text { IncFIBK, IncFII }\end{array}$ & inc 3 & catA2^, sulll & $\begin{array}{l}\text { anrS1, tetA^, } \\
\text { blaTEM-30^ }\end{array}$ \\
\hline $\begin{array}{l}1675479 \\
\text { Laos, } 2015 \\
\text { ST945-2LV, KL125 }\end{array}$ & $\begin{array}{l}\text { p1675479_1 (MK649828) } \\
\text { 167,992 bp } \\
\text { IncFIBK, IncFII }\end{array}$ & inc 3 & $\operatorname{str} A \wedge B$, sulll & tet $A$ \\
\hline $\begin{array}{l}\text { 130411-38618 } \\
\text { Vietnam, } 2011 \\
\text { ST17, KL127 }\end{array}$ & $\begin{array}{l}\text { p130411-38,618_1 (MK649826) } \\
\text { 241,799 bp } \\
\text { IncFIBK, IncFIIK }\end{array}$ & iuc3 & 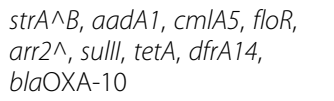 & \\
\hline
\end{tabular}

*rep types as per the PlasmidFinder v2.0 database are shown. All plasmids carried the iuc aerobactin synthesis operon. No plasmids carried the iro or $r m p A / r m p A 2$ virulence loci. Seven virulence plasmids also carried AMR (antimicrobial resistance) genes. Seven host isolates harboured additional AMR genes elsewhere in the genome. ST chromosomal multi-locus sequence type, KL K-locus. ^Inexact match 


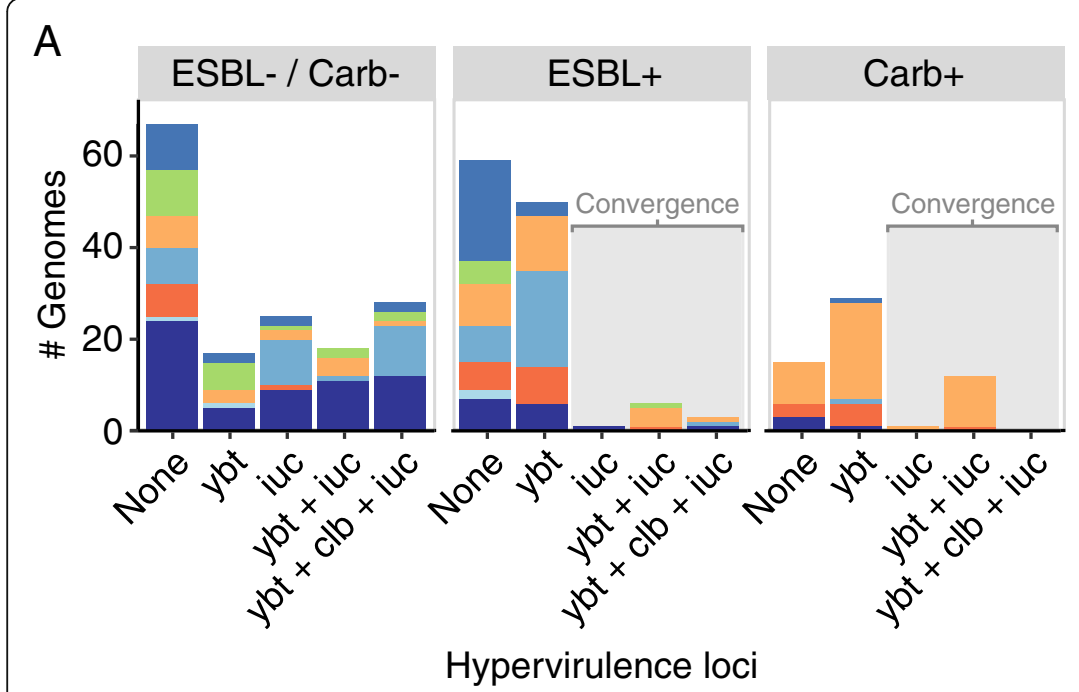

B
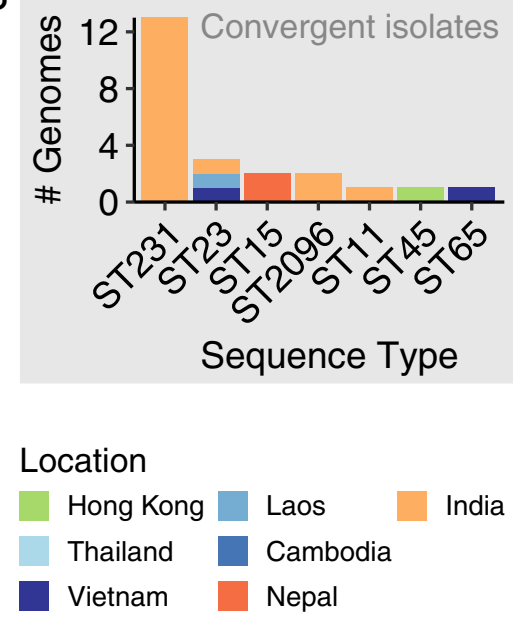

Fig. 5 Convergence of virulence and antimicrobial resistance determinants. a Frequency of genomes carrying the yersiniabactin (ybt), colibactin (clb) and/or aerobactin (iuc) loci shown by ESBL and carbapenemase gene status. Bars are coloured by location as per legend. Grey shading indicates convergent isolates, i.e. those harbouring at least one ESBL and/or carbapenamase gene plus iuc with/without ybt and clb. b Chromosomal sequence types of convergent isolates. Bars are coloured by location as per legend

plus five AMR genes) contained a complete tra operon suggesting that they are capable of conjugative transfer. Consistently, we detected a high degree of sequence similarity between the iuc3 plasmids carried by isolates of three distinct STs in Laos (p1675474 vs p16114547, > 99.9\% nucleotide identity, > 89\% coverage; p1675479 vs p1675474 and p1675479 vs p16114547, > 99\% identity, $>59 \%$ coverage), supporting their dissemination within the local $K$. pneumoniae population or acquisition from a recent common source.

Comparison of chromosomal ST, iuc lineage, and AMR gene content indicates at least nine distinct AMRvirulence convergence events in our sample (Table 3). These include four distinct AMR element acquisitions by previously described hypervirulent STs (three in ST23 from Vietnam, Laos and India; one in ST65 from Vietnam), four distinct virulence plasmid acquisitions by previously described MDR STs (one in ST15 from Nepal; one in ST11 from India; one in ST2096 from India; one in ST231 from India; plus one in ST45 from Hong Kong). Notably, most of these organisms also harboured the additional virulence factors $y b t$, iro and $r m p A / r m p A 2$ (Table 3), supporting the interpretation that they are likely to manifest the hypervirulent phenotype. Three of the strains resulting from these AMRvirulence convergence events showed evidence of local clonal expansion: ST15 in Nepal $(n=2$ isolates, 32 pairwise SNVs in an alignment of 5,709,381 bp, accounting for $\geq 99.5 \%$ of each genome), ST2096 in India ( $n=2$ isolates, 57 pairwise SNVs in an alignment of 5,489,606 bp, accounting for $\geq 96.2 \%$ of each genome) and ST231 in India $(n=13$ isolates, 9 to 319 pairwise SNVs, mean
139.4 SNVs, mean alignment length $5,276,478 \mathrm{bp}$, accounting for mean $96.5 \%$ of each genome). The latter included two potential nosocomial transmission clusters $(\leq$ 25 pairwise SNVs; $n=4$ isolates carrying $i u c 5+b l a_{\text {OXA-232 }}$ with/without $b l a_{\text {CTX-M-15 }} ; n=2$ isolates carrying $i u c 5+$ blaCTX-M-15, see Additional file 1: Table S1).

Each AMR-hypervirulent strain was detected only at one site, with no evidence of dissemination between countries. We did identify eight clusters of closely related $K$. pneumoniae strains (each separated by $\leq 100$ SNVs, in a mean alignment length of $5,435,515 \mathrm{bp}$, accounting for mean $98.1 \%$ of each genome) that were detected in multiple countries, suggesting that regional spread of both MDR and hypervirulent $K$. pneumoniae does occur between Asian countries, as was recently described for CP K. pneumoniae in Europe [70]. However, while most of these clusters were either MDR or hypervirulent, none were both (Table 5). Five clusters were indicative of regional spread between SE Asian countries (ST101, ST17, ST65, ST86 and ST592); the other three clusters were detected in India plus either Laos (ST412), Hong Kong (ST14) or Nepal (ST15) (see Table 5 and Additional file 1: Table S1).

\section{Discussion}

This work represents the first broad genomic study of $K$. pneumoniae causing BSI in S/SE Asia and Hong Kong, regions that are facing a combination of communityacquired invasive hypervirulent $K$. pneumoniae, unregulated use of antimicrobials, and the emergence and spread of MDR pathogens. 
Table 5 Multi-country clusters of strains sharing a recent common ancestor

\begin{tabular}{|c|c|c|c|c|c|c|c|}
\hline ST & Pairwise SNVs (mean) ${ }^{a}$ & Locations & Sample ID & Year & ESBL/CP genes & ICEKp & Plasmid-borne virulence loci \\
\hline \multirow[t]{2}{*}{ ST17 } & 33 & Cambodia & COMRU-KPN-BC-2014-21 & 2014 & - & Unk & - \\
\hline & & Vietnam & CM3425 & 2015 & - & Unk & - \\
\hline \multirow[t]{4}{*}{ ST65 } & $37-138^{b}(89)$ & Laos & 1675485 & 2015 & - & ICEKp $10^{d}$ & iro 1, iuc1, rmpA, rmpA2 \\
\hline & & Laos & 1675477 & 2015 & - & ICEKp 10 $0^{d}$ & iro 1, iuc1, rmpA, rmpA2 \\
\hline & & Laos & 16114200 & 2016 & - & - & iro 1, iuc1, rmpA, rmpA2 \\
\hline & & Cambodia & COMRU-KPN-BC-2015-7 & 2015 & - & ICEKp $10^{d}$ & iro 1, iuc1, rmpA, rmpA2 \\
\hline \multirow[t]{2}{*}{ ST86 } & 69 & Vietnam & $270210-16361$ & 2010 & - & ICEKp4 & iro 1, iuc1, rmpA, rmpA2 \\
\hline & & Laos & 1623415 & 2016 & - & ICEKp4 & irol, iucl, rmpA \\
\hline \multirow[t]{2}{*}{ ST101 } & 73 & Cambodia & KPN239 & 2014 & CTX-M-15 & - & - \\
\hline & & Laos & 1690095 & 2015 & CTX-M-15, CTX-M-27 & - & - \\
\hline \multirow[t]{3}{*}{ ST592 } & 70-91 (80) & Laos & 1675487 & 2015 & - & & iucl, irol, rmpA2 \\
\hline & & Laos & 1675478 & 2015 & - & - & iuc1, iro 1, rmpA2 \\
\hline & & Vietnam & CM3560 & 2015 & - & - & iucl, irol, rmpA2 \\
\hline \multirow[t]{2}{*}{ ST14 } & 82 & Hong Kong & V28 & 2016 & - & - & - \\
\hline & & India & BA33569 & 2016 & - & - & - \\
\hline \multirow[t]{7}{*}{ ST15 } & $0-202(84)^{c}$ & Nepal & 2427 & 2016 & CTX-M-15, OXA-232, NDM-1 & ICEKp 12 & - \\
\hline & & Nepal & 2455 & 2016 & CTX-M-15, OXA-232, NDM-1 & - & - \\
\hline & & Nepal & 2703 & 2016 & CTX-M-15, OXA-232, NDM-1 & - & - \\
\hline & & Nepal & 2725 & 2016 & CTX-M-15, OXA-232, NDM-1 & - & - \\
\hline & & Nepal & 2557 & 2016 & CTX-M-15 & - & iucl, rmpA2 \\
\hline & & Nepal & 2579 & 2016 & CTX-M-15, OXA-232 & - & iuc1, rmpA2 \\
\hline & & India & BA12537 & 2017 & CTX-M-15 & ICEKp12 & - \\
\hline \multirow[t]{2}{*}{ ST412 } & 86 & Laos & 16112578 & 2016 & - & - & iuc1, iro 1, rmpA, rmpA2 \\
\hline & & India & BA2641 & 2017 & - & - & iuc1, iro 1, rmpA, rmpA2 \\
\hline
\end{tabular}

Genomes were clustered at a threshold of $\leq 100$ SNVs (i.e. all members of a cluster differ from at least one other member by fewer than 100 SNVs). Clusters comprising isolates from multiple countries are shown. Unk unknown. ${ }^{a}$ Pairwise SNV count; for clusters of size $>2$ genomes the range and mean are shown. ${ }^{b}$ Cambodian isolate COMRU-KPN-BC-2015-7 differs from each of the Laos isolates by 67-96 SNVs. 'Indian isolate BA12537 differs from each of the Nepalese isolates by $81-202$ SNVs. ${ }^{d}$ ICEKp10 carries ybt and $c l b$

The diversity of $K$. pneumoniae causing BSI in S/SE Asia represents a significant challenge to therapy using existing or novel agents. Our study revealed a highly diverse set of isolates, both in terms of chromosomal STs and surface polysaccharide loci. We were unable to stratify the isolates into hospital-associated and community-acquired cohorts, but anticipate that the former accounts for the majority of the diversity in our sample given that the majority of community-acquired K. pneumoniae BSIs are known to be caused by a small number of distinct clones with limited surface antigen diversity [38-40]. This is consistent with the hypothesis that the majority of hospital-associated BSIs originate from the patients' personal gastrointestinal microbiota rather than from intra-hospital transmissions [67, 79]. The latter is highly relevant to the design of vaccines or other interventions targeting $K$. pneumoniae capsules or lipopolysaccharide, which are considered of high importance in response to increasing prevalence of ESBL and $\mathrm{CP}$ strains, both of which were common in our sampling locations ( $47 \%$ and $17 \%$ of all isolates, respectively). From our sampling strategy, we crudely estimate a noncross-reactive capsule-targeted vaccine would need to include $\geq 16$ serotypes in order to provide potential protection against $>50 \%$ of the BSI $K$. pneumoniae isolates across these study sites. The 16 most common K-loci would cover $61 \%$ of all ESBL and $68 \%$ of all CP K. pneumoniae across S/SE Asia. Alternatively, we estimate that a completely immunising vaccine targeting $\mathrm{O} 1, \mathrm{O} 2$ and O3b would hypothetically protect against $79 \%$ of the BSI K. pneumoniae isolates in this study, equating to $79 \%$ of all ESBL-containing isolates and $70 \%$ of $\mathrm{CP}$.

Alongside the high prevalence of ESBL and carbapenemase genes, our data revealed high prevalence of known hypervirulence determinants with the iuc locus detected in $28 \%$ of all isolates included in this study, more than double the prevalence seen in previous studies focused outside of this region [78]. We suggest this likely reflects the combination of a comparatively greater prevalence of strains from community-acquired infections (particularly 
in SE Asia) plus comparatively elevated prevalence among MDR hospital-associated strains. Accordingly, 33 of 210 (16\%) genomes predicted to be MDR also carried iuc (note includes 5 genomes that were MDR variants of known hypervirulent clones). While the iuc locus itself was common in both S and SE Asia, the distribution of iuc lineages differed (Table 1). Specifically, iuc1 (associated with the characteristic KpVp-1 virulence plasmid [78]) and iuc3 were more common in SE Asia where they were each detected among numerous distinct STs. This is consistent with their local dissemination or recent acquisition from a common source, a finding supported by the discovery of four novel but similar iuc3 encoding plasmids in these isolates. Of greatest concern was the detection of at least nine distinct convergence events between AMR and hypervirulence (encompassing 7.3\% of isolates) plus seven novel dual AMR + iuc containing plasmids, increasing the total number of convergent plasmids reported so far by almost $50 \%[49,80-83]$. Given that our isolate collection represented a snapshot from seven diagnostic laboratories, we predict that many additional convergent plasmid variants await future identification.

It remains unclear if convergent MDR-virulent $K$. pneumoniae plasmids and/or strains are fit for widespread dissemination or simply represent transient events that are rapidly purged from the population. The latter could explain the historical absence of reports of MDR hypervirulent infections and is consistent with the apparent lack of dissemination of any of the numerous reported convergent MDR ST23 variants, despite ample evidence that the susceptible hypervirulent ST23 clone is capable of global distribution [39, 40, 49, 84-87]. However, it is important to note that other factors have also been proposed; for example, the possibility that hypervirulent and MDR clones preferentially inhabit distinct ecological niches outside of the human host, thereby limiting the opportunity for transfer of genetic material between them; or that there may be physical and/or mechanistic barriers to genetic exchange [88]. Additionally, there is now clear evidence that at least a subset of the globally recognised MDR clones with acquired virulence plasmids are able to cause persistent local problems and spread more broadly: These include the $\mathrm{CP}$ ST231 iuc + strain reported here from the Indian hospital, a previously reported CP ST15 $i u c+$ in a Pakistani hospital [53] and CP ST11 iuc + in China, which was able to disseminate for several years without detection $[52,57]$. Fortunately, most MDR-virulent infections thus far appear to be hospital-associated rather than community-acquired, suggesting that unlike the hypervirulent clones, these convergent strains may be less able to spread outside of the hospital environment and/or unable to cause invasive disease in otherwise healthy hosts. Nevertheless, the combination of enhanced virulence plus limited treatment options is an important concern that warrants careful attention. Combined with our data indicating high prevalence and diversity of convergent strains in the S/SE Asian region, it is clear that there is a need for enhanced $K$. pneumoniae surveillance to rapidly identify and monitor convergent strains and/or plasmids.

A limitation of this work is that the data represents a retrospective convenience sample of $K$. pneumoniae BSI isolates by the participating diagnostic laboratories during routine activities during overlapping time periods. This prevented statistical comparisons between individual sites and should be considered when extrapolating gene prevalence information to the broader $K$. pneumoniae population. Additionally, we did not have access to patient clinical data, which has limited our interpretation of the data in the context of patient co-morbidities, and stratification of isolates into community-acquired and hospital-associated cohorts. We focussed on BSI, as this allowed for convenient and consistent retrospective identification of isolates associated with invasive disease for inclusion in the study. While this presentation arguably reflects the greatest clinical need, the siderophore virulence loci are known to be more prevalent in BSI compared to other infections [35], and hence the prevalence of $i u c$ estimated from our sample may be higher than that of the broader population of $K$. pneumoniae causing infections in these regions. In addition, we note that our inferences about AMR are based on genotypic information, which is highly predictive of AMR in $K$. pneumoniae but not perfectly correlated $[67,89]$. Nevertheless, our analyses reveal valuable insights and provide essential data to motivate enhanced public health surveillance.

\section{Conclusions}

K. pneumoniae BSI isolates from South and Southeast Asia represent diverse STs, capsule and LPS serotypes, with high prevalence of MDR, hypervirulence-associated loci and convergent MDR-virulent strains. Our study represents a blueprint for genomic surveillance of emerging AMR pathogens in this region and we urge the coordination of similar activities internationally. By rapidly detecting resistance and virulence genes in the context of clonal and surface antigen diversity, our approach provides critical information that can be used to simultaneously track the emergence and dissemination of clinically important variants, guide antimicrobial therapy and assess potential mechanisms and targets for containment and intervention. In combination with rapid reporting and data sharing, this approach will permit researchers and public health professionals to recognise and control the growing public health threat of AMR $K$. pneumoniae. 


\section{Additional files}

Additional file 1: Table S1. Sample information and genotyping results for all BSI Kp included in this study.

Additional file 2: Table S2. Characteristics of non-Kp BSI isolate genomes. Table S3. K-locus prevalence among K. pneumoniae sensu stricto. Table S4. Predicted O type prevalence among K. pneumoniae sensu stricto.

\section{Acknowledgements}

We would like to acknowledge and thank all staff involved in obtaining and processing the isolates including healthcare facility and laboratory staff at Patan Hospital, Kathmandu, Nepal; Christian Medical College Hospital, Vellore, India; Angkor Hospital for Children, Siem Reap, Cambodia; Mahosot Hospital, Vientiane, Lao PDR; The Hospital of Tropical Diseases, Ho Chi Minh City, Vietnam; Prince of Wales Hospital, Hong Kong; and Shoklo Malaria Research Unit, Thailand. We also thank the WSI Pathogen Informatics team for help with data management.

\section{Funding}

This work was supported by the Wellcome Trust (grant \#206194 to Wellcome Sanger Institute) and the Bill and Melinda Gates Foundation, Seattle (grant \#OPP1175797 to KEH). KEH is supported by a Senior Medical Research Fellowship from the Viertel Foundation of Australia. DAB and PNN are supported by the Wellcome Trust.

\section{Availability of data and materials}

Short-read sequence data have been deposited in the European Nucleotide Archive (www.ebi.ac.uk/ena) under Project accessions PRJEB21605, PRJEB26814 and PRJEB30913. Novel K-locus and plasmid sequences have been deposited in GenBank (www.ncbi.nlm.nih.gov/) under accessions MK593451-MK593454 and MK649822-MK649829, respectively. Isolate information, genotypes and individual genome data accessions are listed in Additional file 1: Table S1. De novo genome assemblies and copies of the K-locus and plasmid sequences are also available in Figshare [72]: doi: https://doi.org/10.26180/5c67982956721.

\section{Authors' contributions}

KLW, NRT, SB and KEH conceived the study. DABD, MI, AK, CLL, TM, PNN, LN, AS, PT, BC and MV collected, identified and collated $K$. pneumoniae isolates. TNTN and LMJ generated genome sequence data. KLW, MMCL and KEH analysed the data. KLW, SB and KEH wrote the manuscript. All authors read and commented on the manuscript. All authors read and approved the final manuscript.

\section{Ethics approval and consent to participate}

This research was performed under the principles of the Helsinki Declaration. For the purposes of this study, all data were collected as part of the routine diagnostic services from each of the collaborating organisations and no human clinical data were accessed or linked with the microbiological data. Consequently, the IRB granted a waiver of ethical approval because $K$. pneumoniae isolates from blood cultures derived from hospital- and/or community-associated BSI following routine diagnostic protocols in each hospital laboratory (identified using biochemical testing, typically API-20E, bioMerieux, or by MALDI-TOF bacterial identification systems) were included in the study.

\section{Consent for publication}

Not applicable.

\section{Competing interests}

The authors declare that they have no competing interests.

\section{Publisher's Note}

Springer Nature remains neutral with regard to jurisdictional claims in published maps and institutional affiliations.

\section{Author details}

'Department of Infectious Diseases, Central Clinical School, Monash University, Melbourne, Victoria 3004, Australia. ${ }^{2}$ Hospital of Tropical Diseases,
Oxford University Clinical Research Unit, Ho Chi Minh City, Vietnam. ${ }^{3}$ The Hospital of Tropical Diseases, Ho Chi Minh City, Vietnam.

${ }^{4}$ Lao-Oxford-Mahosot Hospital-Wellcome Trust Research Unit, Microbiology Laboratory, Mahosot Hospital, Vientiane, Lao People's Democratic Republic

${ }^{5}$ Centre for Tropical Medicine and Global Health, University of Oxford,

Oxford, UK. ${ }^{6}$ London School of Hygiene and Tropical Medicine, London, UK

${ }^{7}$ Department of Microbiology, The Chinese University of Hong Kong, Hong Kong Special Administrative Region, China. ${ }^{8}$ Patan Academy of Health

Sciences, Oxford University Clinical Research Unit, Kathmandu, Nepal. ${ }^{9}$ Shoklo Malaria Research Unit, Mahidol-Oxford Tropical Medicine Research Unit, Faculty of Tropical Medicine, Mahidol University, Mae Sot 63110, Thailand. ${ }^{10}$ Cambodia Oxford Medical Research Unit, Angkor Hospital for Children, Siem Reap, Cambodia. ${ }^{11}$ Department of Clinical Microbiology, Christian Medical College, Vellore, Tamil Nadu, India. ${ }^{12}$ Wellcome Trust Sanger Institute, Hinxton, Cambridge, UK. ${ }^{13}$ Cambridge Institute of Therapeutic Immunology \& Infectious Disease (CITIID) Department of Medicine, University of Cambridge, Cambridge Biomedical Campus, Cambridge CB2 OAW, UK.

Received: 26 July 2019 Accepted: 12 December 2019

Published online: 16 January 2020

\section{References}

1. World Health Organization. Global priority list of antibiotic-resistant bacteria to guide research, discovery, and devlopment of new antibiotics. 2017.

2. Rodriguez-Baño J, Gutiérrez-Gutiérrez B, Machuca I, Pascual A. Treatment of infections caused by extended-spectrum-beta-lactamase-, AmpC-, and carbapenemase-producing Enterobacteriaceae. Clin Microbiol Rev. 2018;31: e00079-17.

3. Rello J, Kalwaje Eshwara V, Lagunes L, Alves J, Wunderink RG, ConwayMorris A, et al. A global priority list of the TOp TEn resistant microorganisms (TOTEM) study at intensive care: a prioritization exercise based on multicriteria decision analysis. Eur J Clin Microbiol Infect Dis. 2018;38:319-23.

4. Dat VQ, Vu HN, Nguyen The H, Nguyen HT, Hoang LB, Vu Tien Viet D, et al. Bacterial bloodstream infections in a tertiary infectious diseases hospital in northern Vietnam: aetiology, drug resistance, and treatment outcome. BMC Infect Dis. 2017;17:493

5. Fox-Lewis A, Takata J, Miliya T, Lubell Y, Soeng S, Sar P, et al. Antimicrobial resistance in invasive bacterial infections in hospitalized children, Cambodia, 2007-2016. Emerg Infect Dis. 2018;24:841-51.

6. Anderson M, Luangxay K, Sisouk K, Vorlasan L, Soumphonphakdy B, Sengmouang $V$, et al. Epidemiology of bacteremia in young hospitalized infants in Vientiane, Laos, 2000-2011. J Trop Pediatr. 2014;60:10-6.

7. The HC, Karkey A, Thanh DP, Boinett CJ, Cain AK, Ellington M, et al. A highresolution genomic analysis of multidrug- resistant hospital outbreaks of Klebsiella pneumoniae. EMBO Molec Med. 2015;7:227-39.

8. Hsu LY, Tan TY, Jureen R, Koh TH, Krishnan P, Lin RTP, et al. Antimicrobial drug resistance in Singapore hospitals. Emerg Infect Dis. 2007;13:1944-7.

9. Hamzan NI, Yean CY, Rahman RA, Hasan H, Rahman ZA. Detection of blaIMP4 and blaNDM1 harboring Klebsiella pneumoniae isolates in a university hospital in Malaysia. Emerg Health Threats J. 2015;8:8-12.

10. Jajoo M, Manchanda V, Chaurasia S, Sankar MJ, Gautam H, Agarwal R, et al. Alarming rates of antimicrobial resistance and fungal sepsis in outborn neonates in North India. PLoS One. 2018;13:e0180705.

11. Mohanty S, Gajanand M, Gaind R. Identification of carbapenemase-mediated resistance among Enterobacteriaceae bloodstream isolates: a molecular study from India. Indian J Med Microbiol. 2017;35:421.

12. Smit $\mathrm{P}$, Stoesser $\mathrm{N}$, Pol $\mathrm{S}$, van Kleef $\mathrm{E}$, Oonsilvilai $\mathrm{M}$, Tan $\mathrm{P}$, et al. Transmission dynamics of hyper-endemic multi-drug resistant Klebsiella pneumoniae in a Southeast Asian neonatal unit: a longitudinal study with whole genome sequencing. Front Microbiol. 2018;9:1197.

13. Runcharoen C, Moradigaravand D, Blane B, Paksanont S, Thammachote J, Anun $S$, et al. Whole genome sequencing reveals high-resolution epidemiological links between clinical and environmental Klebsiella pneumoniae. Genome Med. 2017;9:6.

14. Castanheira M, Deshpande LM, Mathai D, Bell JM, Jones RN, Mendes RE. Early dissemination of NDM-1- and OXA-181-producing Enterobacteriaceae in Indian hospitals: report from the SENTRY antimicrobial surveillance program, 2006-2007. Antimicrob Agents Chemother. 2011;55:1274-8.

15. Tada T, Tsuchiya M, Shimada K, Nga TT, Thu LTA, Phu TT, et al. Dissemination of carbapenem-resistant Klebsiella pneumoniae clinical isolates with various combinations of carbapenemases (KPC-2, NDM-1, NDM-4, and 
OXA-48) and 165 rRNA methylases (RmtB and RmtC) in Vietnam. BMC Infect Dis. 2017:17:467.

16. Laolerd W, Akeda Y, Preeyanon L, Ratthawongjirakul P, Santanirand P. Carbapenemase-producing carbapenem-resistant Enterobacteriaceae from Bangkok, Thailand, and their detection by the Carba NP and modified Carbapenem inactivation method tests. Microb Drug Resist. 2018;24:1006-11.

17. Shankar C, Veeraraghavan B, Nabarro LEB, Ravi R, Ragupathi NKD, Rupali P. Whole genome analysis of hypervirulent Klebsiella pneumoniae isolates from community and hospital acquired bloodstream infection. BMC Microbiol. 2018;18:6.

18. Magiorakos A, Srinivasan A, Carey RB, Carmeli Y, Falagas ME, Giske CG, et al. Multidrug-resistant, extensively drug-resistant and pandrug-resistant bacteria: an international expert proposal for interim standard definitions for acquired resistance. Clin Microbiol Infect. 2012;18:268-81.

19. Shankar C, Shankar BA, Manesh A, Veeraraghavan B. KPC-2 producing ST101 Klebsiella pneumoniae from bloodstream infection in India. J Med Microbiol. 2018;67(7):927-30. https://doi.org/10.1099/jmm.0.000767. Epub 2018 May 22.

20. Shankar C, Kumar S, Venkatesan M, Veeraraghavan B. Emergence of ST147 Klebsiella pneumoniae carrying blaNDM-7 on InCA/C2 with ompK35 and ompK36 mutations in India. J Infect Public Health. 2019;12:741-3.

21. Shankar C, Nabarro LEB, Ragupathi NKD, Sethuvel DPM, Daniel JLK, Doss CGP, et al. Draft genome sequences of three hypervirulent carbapenemresistant Klebsiella pneumoniae isolates from bacteremia. Genome Announc. 2016:4:e01081-16.

22. Pragasam AK, Shankar C, Veeraraghavan B, Biswas I, Nabarro LEB, Inbanathan FY, et al. Molecular mechanisms of colistin resistance in Klebsiella pneumoniae causing bacteremia from India-a first report. Front Microbiol. 2017:7:2135.

23. Mukherjee $S$, Bhattacharjee A, Naha S, Majumdar T. Molecular characterization of NDM-1-producing Klebsiella pneumoniae ST29, ST347, ST1224, and ST2558 causing sepsis in neonates in a tertiary care hospital of north-East India. Infect Genet Evol. 2019:69:166-75.

24. Shankar C, Santhanam S, Kumar M, Gupta V, Devanga Ragupathi NK, Veeraraghavan B. Draft genome sequence of an extended-spectrum- $\beta$-lactamasepositive hypenirulent Klebsiella pneumoniae strain with novel sequence type 2318 isolated from a neonate. Genome Announc. 2016;: :01273-16.

25. Luk S, Wong WK, Ho AY, Yu KC, WK T, Ng TK. Clinical features and molecular epidemiology of plasmid-mediated DHA-type AmpC beta-lactamaseproducing Klebsiella pneumoniae blood culture isolates, Hong Kong. J Glob Antimicrob Resist. 2016:7:37-42.

26. Berglund B, NTB H, Tarnberg M, Le NK, Welander J, Nilsson M, et al. Colistinand carbapenem-resistant Klebsiella pneumoniae carrying $\mathrm{mcr}-1$ and blaOXA48 isolated at a paediatric hospital in Vietnam. J Antimicrob Chemother. 2018:73:1100-2.

27. Van Aartsen JJ, Moore CE, Parry CM, Turner P, Phot N, Mao S, et al. Epidemiology of paediatric gastrointestinal colonisation by extended spectrum cephalosporin-resistant Escherichia coli and Klebsiella pneumoniae isolates in north-west Cambodia. BMC Microbiol BMC Microbiol. 2019:19:1-14.

28. Wyres KL, Wick RR, Gorrie C, Jenney A, Follador R, Thomson NR, et al. Identification of Klebsiella capsule synthesis loci from whole genome data. Microb Genomics. 2016;2

29. Hansen DS, Skov R, Benedí JV, Sperling V, Kolmos HJ. Klebsiella typing: pulsed-field gel electrophoresis (PFGE) in comparison with O:K-serotyping. Clin Microbiol Infect. 2002:8:397-404.

30. Martin RM, Bachman MA. Colonization, infection, and the accessory genome of Klebsiella pneumoniae. Front Cell Infect Microbiol. 2018;8:4.

31. Cheng HY, Chen YS, Wu CY, Chang HY, Lai YC, Peng HL. RmpA regulation of capsular polysaccharide biosynthesis in Klebsiella pneumoniae CG43. J Bacteriol. 2010;192:3144-58.

32. Russo TA, Olson R, MacDonald U, Metzger D, Maltese LM, Drake EJ, et al. Aerobactin mediates virulence and accounts for increased siderophore production under iron-limiting conditions by hypervirulent (hypermucoviscous) Klebsiella pneumoniae. Infect Immun. 2014;82:2356-67.

33. Holden V, Breen P. Houle S, Dozois C, Bachman MA. Klebsiella pneumoniae siderophores induce inflammation, bacterial dissemination, and HIF-1a stabilization during pneumonia. mBio. 2016;7(5). https://doi.org/10.1128/ mBio.01397-16.

34. Lu M-C, Chen Y-T, Chiang M-K, Wang Y-C, Hsiao P-Y, Huang Y-J, et al. Colibactin contributes to the hypervirulence of pks+ K1 CC23 Klebsiella pneumoniae in mouse meningitis infections. Front Cell Infect Microbiol. 2017:7:103.
35. Holt KE, Wertheim H, Zadoks RN, Baker S, Whitehouse CA, Dance D, et al. Genomic analysis of diversity, population structure, virulence, and antimicrobial resistance in Klebsiella pneumoniae, an urgent threat to public health. Proc Natl Acad Sci U S A. 2015;112:E3574-81.

36. Lam MMC, Wick RR, Wyres KL, Gorrie C, Judd M, Brisse S, et al. Genetic diversity, mobilisation and spread of the yersiniabactin-encoding mobile element ICEKp in Klebsiella pneumoniae populations. Microb Genomics. 2018;4

37. Russo TA, Olson R, Fang C-T, Stoesser N, Miller M, MacDonald U, et al. Identification of biomarkers for differentiation of hypervirulent Klebsiella pneumoniae from classical K. pneumoniae. J Clin Microbiol. 2018;56:e00776-18.

38. Siu LK, Yeh KM, Lin JC, Fung CP, Chang FY. Klebsiella pneumoniae liver abscess: a new invasive syndrome. Lancet Infect Dis. 2012;12:881-5.

39. Siu LK, Fung CP, Chang FY, Lee N, Yeh KM, Koh TH, et al. Molecular typing and virulence analysis of serotype K1 Klebsiella pneumoniae strains isolated from liver abscess patients and stool samples from noninfectious subjects in Hong Kong, Singapore, and Taiwan. J Clin Microbiol. 2011:49:3761-5.

40. Lin J-CC, Koh TH, Lee N, Fung C-PP, Chang F-YY, Tsai Y-KK, et al. Genotypes and virulence in serotype K2 Klebsiella pneumoniae from liver abscess and non-infectious carriers in Hong Kong, Singapore and Taiwan. Gut Pathog. 2014;6:21.

41. Wu KM, Li NH, Yan JJ, Tsao N, Liao TL, Tsai HC, et al. Genome sequencing and comparative analysis of Klebsiella pneumoniae NTUH-K2044, a strain causing liver abscess and meningitis. J Bacteriol. 2009;191:4492-501.

42. Chen YT, Chang HY, Lai YC, Pan CC, Tsai SF, Peng HL. Sequencing and analysis of the large virulence plasmid pLVPK of Klebsiella pneumoniae CG43. Gene. 2004;337:189-98.

43. Russo TA, Olson R, MacDonald U, Beanan J, Davidsona BA. Aerobactin, but not yersiniabactin, salmochelin, or enterobactin, enables the growth/survival of hypervirulent (hypermucoviscous) Klebsiella pneumoniae ex vivo and in vivo. Infect Immun. 2015;83:3325-33.

44. Bulger J, MacDonald U, Olsen R, Beanan J, Russo TA. Metabolite transporter PEG344 is required for full virulence of hypervirulent Klebsiella pneumoniae strain hvKp1 after pulmonary but not subcutaneous challenge. Infect Immun. 2017;85:e00093-17.

45. Tu YC, Lu MC, Chiang MK, Huang SP, Peng HL, Chang HY, et al. Genetic requirements for Klebsiella pneumoniae-induced liver abscess in an oral infection model. Infect Immun. 2009;77:2657-71.

46. Russo TA, Marr CM. Hyperviulent Klebsiella pneumoniae. Clin Microbiol Rev. 2019:32:e00001-19.

47. Liu Y, Long D, Xiang T, Du F, Wei DD, Wan L. Whole genome assembly and functional portrait of hypervirulent extensively drug-resistant NDM-1 and KPC-2 co-producing Klebsiella pneumoniae of capsular serotype K2 and ST86. J Antimicrob Chemother. 2019;74:1233-40.

48. Surgers L, Boyd A, Girard PM, Arlet G, Decré D. ESBL-producing strain of hypervirulent Klebsiella pneumoniae K2. France Emerg Infect Dis. 2016;22: $1687-8$.

49. Turton JF, Payne Z, Coward A, Hopkins KL, Turton JA, Doumith M, et al. Virulence genes in isolates of Klebsiella pneumoniae from the UK during 2016, including among carbapenemase gene-positive hypervirulent K1-ST23 and 'non-hypervirulent' types ST147, ST15 and ST383. J Med Microbiol. 2017; 67:118-28.

50. Karlsson M, Stanton R, Ansari U, McAllister G, Chan M, Sula E, et al. Identification of a carbapenemase-producing hypervirulent Klebsiella pneumoniae isolate in the United States. Antimicrob Agents Chemother 2019;63:e00519-19.

51. Harada S, Aoki K, Ishii Y, Ohno Y, Nakamura A, Komatsu M, et al. Emergence of IMP-producing hypervirulent Klebsiella pneumoniae carrying a pLVPK-like virulence plasmid. Int J Antimicrob Agents. 2019;53:873-5.

52. Gu D, Dong $N$, Zheng $Z$, Lin $D$, Huang $M$, Wang $L$, et al. A fatal outbreak of ST11 carbapenem-resistant hypervirulent Klebsiella pneumoniae in a Chinese hospital: a molecular epidemiological study. Lancet Infect Dis. 2017;3099:1-10.

53. Heinz E, Ejaz H, Bartholdson Scott J, Wang N, Gujaran S, Pickard D, et al. Resistance mechanisms and population structure of highly drug resistant Klebsiella in Pakistan during the introduction of the carbapenemase NDM-1. Sci Rep. 2019;9:2392

54. Shu L, Dong N, Lu J, Zheng Z, Hu J, Zeng W, et al. Emergence of OXA-232 carbapenemase-producing Klebsiella pneumoniae that carries a pLVPK-like virulence plasmid among elderly patients in China. Antimicrob Agents Chemother. 2019;63:e02246-18. 
55. Caneiras C, Lito L, Melo-cristino J, Duarte A. Community- and hospitalacquired Klebsiella pneumoniae urinary tract infections in Portugal: virulence and antibiotic resistance. Microorganisms. 2019;7:138.

56. Huang $Y$, Chou S, Liang S, Ni C, Lin Y, Huang Y, et al. Emergence of an XDR and carbapenemase-producing hypervirulent Klebsiella pneumoniae strain in Taiwan. J Antimicrob Chemother. 2018;73:2039-46.

57. Du P, Zhang Y, Chen C. Emergence of carbapenem-resistant hypervirulent Klebsiella pneumoniae. Lancet Infect Dis. 2017;3099:30629.

58. Wick RR, Judd LM, Gorrie CL, Holt KE. Completing bacterial genome assemblies with multiplex MinION sequencing. Microb Genomics. 2017;3

59. Bankevich A, Nurk S, Antipov D, Gurevich AA, Dvorkin M, Kulikov AS, et al. SPAdes: a new genome assembly algorithm and its applications to singlecell sequencing. J Comput Biol. 2012;19:455-77.

60. Wick RR, Judd LM, Gorrie CL, Holt KE. Unicycler: resolving bacterial genome assemblies from short and long sequencing reads. PLoS Comp Biol. 2017;13: e1005595.

61. Wick RR, Heinz E, Holt KE, Wyres KL. Kaptive web: user-friendly capsule and lipopolysaccharide serotype prediction for Klebsiella genomes. J Clin Microbiol. 2018;56:e00197-18

62. Pan Y-J, Lin T-L, Chen C-T, Chen Y-Y, Hsieh P-F, Hsu C-R, et al. Genetic analysis of capsular polysaccharide synthesis gene clusters in 79 capsular types of Klebsiella spp. Nat Sci Rep. 2015;5:15573.

63. Follador R, Heinz E, Wyres KL, Ellington MJ, Kowarik M, Holt KE, et al. The diversity of Klebsiella pneumoniae surface polysaccharides. Microb Genomics. $2016 ; 2$

64. Wick RR, Schultz MB, Zobel J, Holt KE. Bandage: interactive visualization of de novo genome assemblies. Bioinformatics. 2015;31:3350-2.

65. Seemann T. Prokka: rapid prokaryotic genome annotation. Bioinformatics. 2014:30:2068-9.

66. Brisse S, Passet V, Haugaard AB, Babosan A, Kassis-Chikhani N, Struve C, et al. wzi gene sequencing, a rapid method for determination of capsular type for Klebsiella strains. J Clin Microbiol. 2013;51:4073-8. [cited 2014 Mar 11]

67. Gorrie CL, Wick RR, Edwards DJ, Strugnell RA, Pratt N, Garlick J, et al. Gastrointestinal carriage is a major reservoir of $K$. pneumoniae infection in intensive care patients. Clin Infect Dis. 2017;65:208-15.

68. Souvorov A, Agarwala R, Lipman DJ. SKESA: strategic K-mer extension for scrupulous assemblies. Genome Biol. 2018;19:153.

69. Sherry NL, Lane CR, Kwong JC, Schultz M, Sait M, Stevens K, et al. Genomics for molecular epidemiology and detecting transmission of carbapenemaseproducing Enterobacterales in Victoria, Australia, 2012-2016. J Clin Microbiol. 2019;57:1-12.

70. David S, Reuter S, Harris SR, Glasner C, Feltwell T, Argimon S, et al. Epidemic of carbapenem-resistant Klebsiella pneumoniae in Europe is driven by nosocomial spread. Nat Microbiol. 2019:4(11):1919-29. https://doi.org/10. 1038/s41564-019-0492-8. Epub 2019 Jul 29.

71. Carattoli A, Zankari E, Garciá-Fernández A, Larsen MV, Lund O, Villa L, et al. PlasmidFinder and PMLST: in silico detection and typing of plasmids. Antimicrob Agents Chemother. 2014;58:3895-903.

72. Wyres KL, Nguyen TNT, Lam MMC, Judd LM, Chau N, van V, DAB D, et al. Data for, "Genomic surveillance for hypervirulence and multi-drug resistance in invasive Klebsiella pneumoniae from south and southeast Asia". Figshare Collection. 2019; https://doi.org/10.26180/5c67982956721.

73. R Development Core Team R. R: A Language and Environment for Statistical Computing. R Found Stat Comput. 2011. https://www.r-project.org.

74. Wickham H. ggplot2: Elegant Graphics for Data Analysis: Springer; 2009.

75. Lin T-L, Lee C-Z, Hsieh P-F, Tsai S-F, Wang J-T. Characterization of integrative and conjugative element ICEKp1-associated genomic heterogeneity in a Klebsiella pneumoniae strain isolated from a primary liver abscess. J Bacteriol. 2008;190:515-26. pmcentrez\&rendertype=abstract

76. Lai YC, Lin AC, Chiang MK, Dai YH, Hsu CC, Lu MC, et al. Genotoxic Klebsiella pneumoniae in Taiwan. PLoS One. 2014:9:e96292.

77. Lam MMC, Wyres KL, Duchêne S, Wick RR, Judd LM, Gan Y, et al. Population genomics of hypervirulent Klebsiella pneumoniae clonal group 23 reveals early emergence and rapid global dissemination. Nat Commun. 2018;9:2703.

78. Lam MCC, Wyres KL, Judd LM, Wick RR, Jenney A, Brisse S, et al. Tracking key virulence loci encoding aerobactin and salmochelin siderophore synthesis in Klebsiella pneumoniae. Genome Med. 2018;10:77.

79. Martin RM, Cao J, Brisse S, Passet V, Wu W, Zhao L, et al. Molecular epidemiology of colonizing and infecting isolates of Klebsiella pneumoniae. mSphere. 2016;1(5). eCollection 2016 Sep-Oct.
80. Dong N, Lin D, Zhang R, Chan EWC, Chen S. Carriage of blaKPC-2 by a virulence plasmid in hypervirulent Klebsiella pneumoniae. J Antimicrob Chemother. 2018;73:3317-21.

81. Lam MMC, Wyres KL, Wick RR, Judd LM, Fostervold A, Holt KE, et al. Convergence of virulence and multidrug resistance in a single plasmid vector in multidrug-resistant Klebsiella pneumoniae ST15. J Antimicrob Chemother. 2019;74:1218-22.

82. Shen D, Ma G, Li C, Jia X, Qin C, Yang T, et al. Emergence of a multidrugresistant hypervirulent Klebsiella pneumoniae of ST23 with a rare blaCTX-M24 -harboring virulence plasmid. Antimicrob Agents Chemother. 2019;63: e02273-18

83. Turton J, Davies F, Turton J, Perry C, Payne Z, Pike R. Hybrid resistance and virulence plasmids in "high-risk" clones of Klebsiella pneumoniae, including those carrying blaNDM-5. Microorganisms. 2019;7:326.

84. Gundestrup S, Struve C, Stahlhut SG, Hansen DS. First case of liver abscess in Scandinavia due to the international hypervirulent Klebsiella pneumoniae clone ST23. Open Microbiol J. 2014;8:22-4.

85. Vila A, Cassata A, Pagella H, Amadio C, Yeh KK-M, Chang FF-Y, et al. Appearance of Klebsiella pneumoniae liver abscess syndrome in Argentina: case report and review of molecular mechanisms of pathogenesis. Open Microbiol J. 2011;5:107-13.

86. Decré D, Verdet C, Emirian A, Le Gourrierec T, Petit JC, Offenstadt G, et al. Emerging severe and fatal infections due to Klebsiella pneumoniae in two university hospitals in France. J Clin Microbiol. 2011:49:3012-4.

87. Hall JM, Hall JM, Ingram PR, Bs MB, Reilly LCO, Inglis TJJ. Temporal flux in beta-lactam resistance among Klebsiella pneumoniae in Western Australia. J Med Microbiol. 2016;65:429-37.

88. Wyres KL, Wick RR, Judd LM, Froumine R, Tokolyi A, Gorrie CL, et al. Distinct evolutionary dynamics of horizontal gene transfer in drug resistant and virulent clones of Klebsiella pneumoniae. PLoS Genet. 2019;15:e1008114.

89. Stoesser N, Batty EM, Eyre DW, Morgan M, Wyllie DH, Del Ojo EC, et al. Predicting antimicrobial susceptibilities for Escherichia coli and Klebsiella pneumoniae isolates using whole genomic sequence data. J Antimicrob Chemother. 2013;68:2234-44
Ready to submit your research? Choose BMC and benefit from:

- fast, convenient online submission

- thorough peer review by experienced researchers in your field

- rapid publication on acceptance

- support for research data, including large and complex data types

- gold Open Access which fosters wider collaboration and increased citations

- maximum visibility for your research: over $100 \mathrm{M}$ website views per year

At BMC, research is always in progress.

Learn more biomedcentral.com/submission 